\title{
A FOXO-dependent replication checkpoint restricts proliferation of damaged cells
}

Marten Hornsveld ${ }^{1,2^{*}}{ }^{*}$ Femke M Feringa ${ }^{3,4^{*}}$, Lenno Krenning ${ }^{3}$, Jeroen van den Berg3,5, Lydia MM Smits², Nguyen BT Nguyen², Maria J Rodriguez-Colman², Tobias B Dansen², Rene H Medema 3 , Boudewijn MT Burgering ${ }^{\ddagger}$

Author affiliations:

${ }^{1}$ Oncode Institute, Department of Cell and Chemical Biology, Leiden University Medical Center, 2333CZ, Leiden, The Netherlands.

2 Oncode Institute, Center for Molecular Medicine, University Medical Center Utrecht, Utrecht University, 3584CG, Utrecht, The Netherlands.

3 Oncode Institute, Division of Cell Biology, Netherlands Cancer Institute, 1066CX, Amsterdam, The Netherlands.

${ }^{4}$ Department of Molecular and Cellular Neurobiology, Faculty of Science, Center for Neurogenomics and Cognitive Research, Amsterdam Neuroscience, Vrije Universiteit Amsterdam, 1081HV, Amsterdam, The Netherlands.

${ }^{5}$ Oncode Institute, Hubrecht Institute-KNAW (Royal Netherlands Academy of Arts and Sciences), 3584CT, Utrecht, The Netherlands.

* These authors contributed equally to this work

${ }^{\ddagger}$ Correspondence can be send to Marten Hornsveld and Boudewijn MT Burgering, e-mail: m.hornsveld@lumc.nl and b.m.t.burgering@umcutrecht. 


\section{Abstract}

DNA replication is challenged by numerous exogenous and endogenous factors that can interfere with the progression of replication forks. Stalling or slowing of the replication fork as a result of replication stress leads to formation of aberrant single-stranded DNA (ssDNA) stretches and potentially DNA double-stranded-breaks (DSBs). Accumulation of ssDNA activates the ATR-dependent DNA replication stress checkpoint response that slows progression from S/G2- to M-phase to protect genomic integrity (1). However, whether mild replication stress restricts proliferation remains controversial (2-6). Here we identify a novel cell cycle exit mechanism, that prevents S/G2 phase arrested cells from undergoing mitosis after exposure to mild replication stress through premature activation of the CDH1 bound Anaphase Promoting Complex / Cyclosome (APC/CCDH1). We find that replication stress causes a gradual decrease of the levels of the APC/CDH1 inhibitor EMI1/FBX05 through Forkhead Box O (FOXOs) mediated repression of its transcriptional regulator E2F1. By doing so, FOXOs limit the time during which the replication stress checkpoint is reversible, and thereby play an important role in maintaining genomic stability.

\section{Results}

Mild replication stress leads to under-replicated DNA which may be tolerated by cells, even though this gives rise to DNA lesions upon mitotic progression $(3,4)$. Indeed, replication stress may induce gaps, breaks and micro-deletions at common fragile sites (CFS)(7,8), highlighting its mutagenic potential. Therefore, it is likely that cells have mechanisms to prevent the propagation of under replicated DNA. To investigate this, we visualized the cellular response to mild replication stress by aphidicolin in nontransformed RPE-1 cells with endogenously tagged Cyclin B1 $1^{\mathrm{YFP}}$ (RPE-CCNB ${ }^{\mathrm{YFP}}$ ) and 
stable expression of 53BP1 mCherry (RPE $\left.C C N B^{\mathrm{YFP}}-53 B P 1^{\mathrm{mCherry}}\right)(9)$ (Fig. 1A). While the addition of aphidicolin had no effect on cells in G2-phase (identified by Cyclin B1 expression), cells that progressed through S-phase in the presence of aphidicolin showed a clear decrease in mitotic entry over time (Fig. 1B). This decrease in mitotic entry was accompanied by a concomitant increase in cells that abruptly lost Cyclin B1 expression (Fig. 1C). Based on previous findings (10) we hypothesized that the loss of Cyclin B1 expression may correspond to levels of DNA damage. Indeed, cells that degrade Cyclin B1 after progressing through S-phase with mild replication stress, display increased 53BP1 foci compared to control cells or cells that recovered from replication stress and progressed to mitosis (Fig. 1D). Importantly, abrogation of DNA damage signaling by ATR inhibition caused an increase in 53BP1 foci number in G1 daughter cells (Fig. S1A).

We and others have previously shown that loss of Cyclin B1 and other cell cycle targets after DNA double-stranded breaks (DSBs) is mediated via APC/C ${ }^{\mathrm{CDH} 1}$-dependent protein-degradation, causing the cells to enter a state of senescence (10-12). To examine whether the aphidicolin-induced loss of Cyclin B1 is caused by APC/CCDH1 activation, we depleted CDH1. Indeed, CDH1 knock-down prevented Cyclin B1 degradation in cells that progressed through S-phase in the presence of aphidicolin (Fig. 1E \& S1B). Experiments using RPE-1 cells stably expressing the Fluorescent Ubiquitination-based Cell Cycle Indicator (FUCCI) probes hCDT1 (aa30/120)-mKO2 (degraded by SCFSKP2 in S/G2/Mphases, thus indicating G1-phase) and hGeminin(aa1-110)-mAG (degraded by APC/CCDH1 in G1-phase, thus indicating S/G2/M-phases) confirmed that APC/C ${ }^{C D H 1}$ activation indeed occured in G2. Aphidicolin treatment of RPE-FUCCI cells resulted in the accumulation of 4n mKO2 ${ }^{+}$cells over time, showing that these cells lost the APC/CCDH1 target Geminin and SCFSKP2 activity without going through mitosis (Fig. 1F). We and others previously demonstrated that the balance between a reversible arrest and the APC/C $\mathrm{CDH}^{\text {-dependent }}$ 
cell cycle exit caused by DNA double strand breaks in G2-phase is be tipped towards cell cycle exit by p53-dependent p21 expression $(10,11)$. Interestingly, we observe that $\mathrm{APC} / \mathrm{C}^{\mathrm{CDH} 1}$ activation in response to aphidicolin is delayed but not abrogated in $\mathrm{p} 21$ $(\Delta \mathrm{p} 21)$ and p53 ( $\Delta$ p53) knockout RPE-FUCCI cells (Fig. 1G).

In G2, activation of APC ${ }^{\mathrm{CDH} 1}$ is prevented by CDK1/2-dependent phosphorylation of CDH1 $(13,14)$. To characterize CDK activity following replication stress, we used the previously described CDK2-activity sensor in RPE $C C N B^{\mathrm{YFP}}$ cells $(9,15)$. We find that CDK2 activity is stable in G2 cells following mild replication stress in S phase and drops in synchrony with Cyclin B1 (Fig. 1H). The simultaneous loss of CDK2 activity and $\mathrm{APC} / \mathrm{C}^{\mathrm{CDH} 1}$ target $\mathrm{Cyclin} \mathrm{B} 1$ is surprising, since $\mathrm{APC} / \mathrm{C}^{\mathrm{CDH} 1}$ activation in response to DNA double strand breaks is described to depend on the premature loss of CDK activity $(10,11,16-19)$. Therefore we expected CDK2 activity to drop before Cyclin B1, yet our results implicate that $\mathrm{APC} / \mathrm{C}^{\mathrm{CDH} 1}$ can be activated despite the present level of $\mathrm{CDK} 2$ activity.

In addition to $\mathrm{CDK} 1 / 2$ mediated inhibition, the $\mathrm{APC} / \mathrm{C} \mathrm{CDH} 1$ inhibitor EMI1 blocks APC/CCDH1 activity during S/G2 and loss of EMI1 is sufficient for premature activation of APC/CCDH1 in G2 $(17,20-23)$. We therefore combined our RPE $C C N B^{\mathrm{YFP}}-53 B P 1^{\text {mCherry }}$ cells with doxycycline inducible EMI1-overexpression (9), to define the importance of EMI1 loss for $\mathrm{APC} / \mathrm{C}^{\mathrm{CDH} 1}$ activation following replication stress. Overexpression of EMI1 prevented Cyclin B1 degradation and rescued mitotic entry of cells exposed to replication stress (Fig. 1I \& S1C). As replication stress results predominantly in ATR/CHK1activating lesions (24), this raises the question whether sustained ATR-signaling is required to maintain cells arrested and induce cell cycle exit. To address this, we treated G2 cells that progressed through S phase in the presence of aphidicolin with the specific ATR inhibitor VE-821. ATR inhibition prevented Cyclin B1 degradation and resulted in 
immediate mitotic entry of almost all G2 cells that progressed through $\mathrm{S}$ phase in presence of aphidicolin (Fig. 1I \& S1C). In contrast, aphidicolin-treated cells that did recover from replication stress in the DMSO-treated condition showed a clear delay in mitotic entry (Fig. 1I). The rescue by ATR inhibition was distinct from the rescue observed upon EMI1 overexpression, since EMI1 expressing G2 cells still arrested for several hours before progressing into mitosis. These results show that sustained ATR activity maintains the $\mathrm{G} 2$ arrest that ultimately results in $\mathrm{APC} / \mathrm{C}^{\mathrm{CDH}} 1$-dependent cell cycle exit. Together, these results imply there are additional players in the replication stress checkpoint that steer the decision between entering mitosis or exiting G2 through regulation of $\mathrm{APC} / \mathrm{C}^{\mathrm{CDH} 1}$ activation. Surprisingly, this regulation is not mediated through p53/p21 or Cyclin B/CDK1 signaling.

DNA damage results in the activation of a transcriptional program that promotes DNA repair and stalls cell cycle progression (25). To determine whether a similar transcriptional program is induced by the canonical replication stress checkpoint, we determined the transcriptome of control and aphidicolin-treated cells. We identified 272 genes that were differentially expressed upon replication stress (Fig. 2A, log2fold $0.5<x>0.5, p<0,05,155$ down- and 117 up-regulated genes). Transcription factor binding sites (TFBS) analysis revealed a significant enrichment of Forkhead Box 0 (FOXO) target genes that were upregulated in response to aphidicolin (Fig. 2A-B). As FOXOs are known regulators of cell cycle arrest and are involved in the DNA damage response $(26,27)$, we determined if FOXOs are activated in response to replication stress. Indeed, we observed an increase in nuclear FOXO3, specifically in S/G2-phase cells already at 2 hours after aphidicolin treatment (Fig. 2C,D). Although FOXOs usually function redundantly and mRNA expression of FOXO1, FOXO3 and FOXO4 increases after aphidicolin treatment, 
FOX04 protein is undetectable in RPE cells (28) and FOXO1 localization was hardly affected by aphidicolin treatment, suggesting a more dominant role for FOXO3 in response to replication stress (Fig. S2A,B). Nuclear translocation of FOX03 was reduced in the presence of ATR inhibitor, showing that ATR somehow influences FOXO3 activation in response to replication stress (Fig. 2D).

To study whether nuclear localization of FOXO is sufficient to cause premature APC/CCDH1 activation, we transduced a doxycycline-inducible, constitutively nuclear mutant of FOXO3 (FOX03.A3) in our RPE-FUCCI cells (Fig. S2C), and analyzed the fate of cells with activated FOXO3 by time lapse microscopy. FOXOs are known to induce a robust G1 cell cycle arrest, and therefore only cells in early S/G2 at the time of FOX03.A3 induction were included in our analysis (29). Upon FOX03.A3 expression cells arrest for 15-35 hours in S/G2-phase before prematurely activating the $\mathrm{APC} / \mathrm{C}^{\mathrm{CDH}} 1$, much alike the cellular response to replication stress (Fig. 2E). Additionally, we find that $74 \pm 4 \%$ of all $4 \mathrm{n}$ cells lost geminin expression (mAG-) and are mKO+ at 30h after doxycycline-induced F0X03.A3 (Fig. 2F-G), indicative of $\mathrm{APC} / \mathrm{C}^{\mathrm{CDH}} 1$ activation and cell cycle exit from G2 without mitosis. FOX03.A3 expression strongly enhances aphidicolin-induced premature APC/CCDH1 activation, both when FOX03.A3 is induced simultaneously with aphidicolin treatment or following $24 \mathrm{~h}$ pre-treatment with aphidicolin, which accumulates cells in G2 prior to FOX03.A3 induction (Fig. 2F,G). Interestingly, we observe that F0X03.A3 expression did not induce p53 or p21 expression and induces APC/CCDH1 activation in S/G2 of RPE-FUCCI-FOX03 cells knockout for p53 ( $\Delta$ p53) or p21 ( $\Delta$ p21) (Fig. 2H \& S3AC).

Collectively, these observations imply that FOXOs promote premature APC/CCDH1 activation in response to replication stress. In order to test whether FOXOs play an essential role in this process, we generated RPE-FUCCI cells with a doxycycline-inducible 
shRNA that efficiently knocks down FOXO1 and FOXO3 (RPE-FUCCI-shFOXOs) (30-32). Aditionally, to separate any FOXO-dependent effect from p53- and p21-mediated effects, we also introduced the inducible shFOXOs into $\Delta$ p21 and $\Delta$ p53 RPE-FUCCI cells. Replication stress induced the expression of p21 in a p53-dependent manner and p53 and p21 expression are reduced in the absence of FOXOs, both in unperturbed conditions and after replication stress (Fig. 3A). While FOXO-dependent p53 activation is not required for replication stress-induced cell cycle exit (Fig. 1G). However, FOXO depletion itself did prevent replication stress-induced premature $A P C / C^{C D H} 1$ activation in $G 2$, uncovering an additional role for FOXO in the cellular response to replication stress (Fig. 3B,C). Loss of FOXOs lowered the activity of CHK1 and CHK2 in response to aphidicolin and other DNA damaging agents like hydroxyurea (HU) and neocarzinostatin (NCS) (Fig. 3D), but did not prevent initial DNA damage signaling, as FOXO-depletion did not significantly reduce the formation of $\gamma \mathrm{H} 2 \mathrm{AX}$ foci in response to aphidicolin treatment (Fig. 3E). Downstream events, such as the loading of mono-ubiquitinated FANCD2 and the establishment of 53BP1 foci in S/G2 cells, following replication stress were perturbed in the absence of FOXO activity (Fig. 3F-H). Together these results delineate an important role for FOXOs in both replication stress checkpoint activation and steering cell fate by stimulating a cell cycle exit following replication stress. This suggests that reducing FOXO expression might increase resistance to aphidicolin treatment. Indeed, loss of FOXOs increased the IC50 of aphidicolin in RPE-FUCCI cells by 54\% (from $113.1 \mathrm{nM}$ in control to $174.1 \mathrm{nM}$ in FOXO-depleted RPE-FUCCI cells)(Fig. 3I). Taken together, we uncover FOXOs as novel important players in the replication stress response but still wonder what is the event downstream of FOXOs nuclear translocation that drives cell cycle withdrawal. 
Our data show that FOXOs can drive APC/CCDH1 activation following replication stress. Indeed, we observed that the FOXO3.A3-induced loss of Geminin expression in RPEFUCCI cells (4n mKO+ cells) is reduced upon CDH1 knock-down (Fig. S4A-C). In addition, F0X03.A3 expression results in a gradual decrease in EMI1 expression in asynchronously cultured cells, which is in line with previous studies showing that EMI1 downregulation is a prerequisite for cell cycle exit in G2 phase (Fig. 4A \& Fig. S4D) $(9,11,17)$. To exclude that EMI1 downregulation is not simply the effect of a FOXO-induced G1 arrest, we sorted RPE-FUCCI-FOX03.A3 cells based on mKO and mAG expression 8 and 16 hours after FOXO3 induction. As expected, EMI1 expression is not detected in G0/G1-phase and high in S/G2-phases in control cells (Fig. 4B,C). Strikingly, FOXO3.A3 induction strongly diminished EMI1 expression in S/G2-phase cells both at the level of mRNA and protein (Fig. 4B-C). Accordingly, RPE-FUCCI cells were more prone to exit the cell cycle when EMI1 knockdown was combined with FOX03.A3 expression (Fig. 4D \& S4E). To determine whether EMI1 expression can prevent FOXO3-induced premature APC/CCDH1 activation, we constructed RPE-FUCCI cells with doxycycline-inducible FOX03.A3 and mTurq2-EMI1 (RPE-FUCCI iFOX03.A3/mTurq2-EMI1) (Fig. 4E). While F0X03.A3 expression reduced mitotic entry of G2 cells due to premature APC/CCDH1 activation (Fig. 4F), simultaneous expression of EMI1 blocked APC/CCDH1 activation in G2, allowing cells to enter mitosis (Fig. 4F). Subsequently, cells arrested at metaphase as a consequence of high EMI1 levels in mitosis (Fig. 4F\& S4F)(33).

We noticed that endogenous EMI1 levels still decreased in FOXO3.A3- and EMI1overexpressing cells (Fig. 4E), suggesting that transcriptional repression by FOXOs is responsible for reduced endogenous EMI1 expression. If FOXOs regulate EMI1 levels in S/G2 phase in response to replication stress, loss of FOXOs should stabilize EMI1 levels. Indeed, aphidicolin-induced loss of EMI1 in S/G2-phase cells is prevented upon FOXO 
depletion (Fig. 4G-H). Hence, we conclude that FOXO-induced APC/CCDH1-activation is mediated via repression of EMI1.

Although $\sim 20 \%$ of all genes in the genome have FOXO binding elements in their promoters, FOXO binding sites are absent directly upstream of EMI1, implying that EMI1 transcription is not suppressed by FOXOs binding to its promoter directly $(34,35)$. The main transcription factor driving EMI1 expression is E2F1 (21), and previous studies have shown that FOXOs can bind to E2F1 and alter E2F1 target gene expression (32). Indeed, co-immunoprecipitation (co-IP) experiments using GFP-FOXO1/3 and HA-E2F1 confirmed that FOXOs can bind to E2F1 (Fig. S5A). To test whether FOXOs indeed affect E2F1-dependent transcription of EMI1 and other genes, we performed Chromatin-IP (ChIP) experiments. We found that binding of endogenous E2F1 to the promoter of EMI1 as well as two other canonical E2F1 targets (Cyclin E (CCNE2) and A (CCNA2)) was reduced when FOX03.A3 was expressed (Fig. 4I). Accordingly, the expression of E2F1 target genes (E2F1 itself, CCNE2 and CCNA2) was reduced (Fig. S5B-D). Indeed, FOXOinduced $\mathrm{APC} / \mathrm{C}^{\mathrm{CDH} 1}$ activation is enhanced when cells enter S-phase with lowered E2F1 levels, as combining E2F1 knockdown with FOXO3.A3 expression sensitizes RPE-FUCCI cells to activate APCCDH1 in S/G2 (Fig. 4J,K \& S6E).

Next, we determined whether cells that activate APC/CCDH1 in G2-phase truly switch to a G0/G1 state after EMI1 downregulation. To this end, we sorted RPE-FUCCIFOX03.A3 cells based on mAG, mKO and DNA content (Hoechst 33342) at 24 hours after FOX03.A3 induction (Fig. 4L). 2n mKO+ cells reflect G1 as EMI1 levels and RB phosphorylation are low, Cyclin D is high and both Cyclin A and B are absent. FOXO3.A3 expression in $2 \mathrm{n} \mathrm{mKO+} \mathrm{cells} \mathrm{induced} \mathrm{expression} \mathrm{of} \mathrm{its} \mathrm{target} \mathrm{p27} \mathrm{and} \mathrm{reduced} \mathrm{the}$ expression of Cyclin D, known to lead to a G1 arrest (29). In 4n mAG+ control cells the expression of EMI1, Cyclin A, Cyclin B, SKP2, CDH1 and phosphorylation of RB are high, 
confirming that these cells are indeed in S/G2-phase. Strikingly, FOXO3 activation reduced EMI1 expression in $4 \mathrm{n} \mathrm{mAG+} \mathrm{cells,} \mathrm{illustrating} \mathrm{that} \mathrm{FOXO} \mathrm{mediated} \mathrm{EMI1}$ repression precedes $\mathrm{APC} / \mathrm{C}^{\mathrm{CDH} 1}$ activation. Additionally, P27 and p21 are not induced, in line with the fact that SCFSKP2 mediates their degradation in S/G2 cells $(36,37)$. Importantly, 4n mKO+ cells resemble G1 cells as expression of EMI1, Cyclin A, Cyclin B, SKP2 and phosphorylation of RB are absent. The high p27 and p21 levels in 4n mKO+ cells suggest that these cells are arrested. Finally, this experiment also confirms our initial observation that $\mathrm{APC} \mathrm{CDH}^{1}$ activation is not preceded by $\mathrm{CDK}$ inhibition as p27 and p21 only increase after the APC/C is activated. Collectively these data show that FOXOdependent premature activation of the APC/C $\mathrm{CDH}^{\mathrm{C} 1}$ prevents mitotic entry and in stead reverts the cells back into a $4 \mathrm{~N}$ G1-like cellular state.

\section{Conclusion \& Discussion}

Here we investigated the cellular response to replication stress, and find that cells monitor the presence of residual damage when progressing from $\mathrm{S}$ to $\mathrm{G} 2$ phase. We identified that a fraction of cells initiates cell cycle exit from G2 phase in response to replication stress. This is in contrast to earlier work that suggested that both transformed and non-transformed cells that experienced replication stress progress through mitosis, resulting in chromosomal aberrations and 53BP1-foci in G1-phase (3-5). In agreement with previous reports, we show that G2 phase can be extended in an ATR-dependent manner, to provide time for DNA repair (5). However, if repair is not successful within $\sim 24$ hours, APC/C $\mathrm{CDH}^{\mathrm{C} 1}$ is activated and cells exit the cell cycle. It has previously been shown that DSBs in G2 phase may cause premature APC/CCDH1-activation, resulting in irreversible withdrawal from the cell cycle $(9-12,17)$. Premature APC/CCDH1-activation is preceded by p21-dependent nuclear entrapment of Cyclin B1-CDK complexes, which 
renders it inert for CDK (re-)activation $(10,18)$. Intriguingly, here we find that CDK2 remains active during a replication stress-induced G2 arrest, and that $\mathrm{APC} / \mathrm{C}^{\mathrm{CDH} 1}$ can be activated independent from p53, p21 or CDK2 inhibition. This leads to the interesting conclusion that $\mathrm{APC} / \mathrm{C}^{\mathrm{CDH}} 1$ can be activated after replication stress in cells containing active CDK-complexes. We find that aphidicolin-induced APC/CCDH1-activation is dependent on the repression of EMI1. FOXOs act as a timer to restrict the window during which cells may recover from replication stress, by gradually decreasing EMI1 levels through removing E2F1 from the EMI1 promoter (Fig. 4M). Alteration of E2F1 dependent transcriptional output by FOXOs has been reported previously for genes involved in apoptosis, and we now show that this mechanism of action is also applicable to cell cycle regulation in S/G2-phase (32).

Interestingly, FOXOs and p53/p21 co-operate to restrict the time during which replication stress induces a reversible cell cycle arrest. On the one hand, FOXOs support the checkpoint and sensitize the APC/CCDH1 to activation, on the other hand p21 inhibits CDK activity. Combined, this results in a robust switch in which APC/CCDH1-activation can revert the cell from a S/G2 state into G0/G1. Next to indirectly regulating the APC/C $\mathrm{CDH}$, we observed that FOXOs are required for the establishment of the replication stress checkpoint. As FOXOs have been implicated to play a similar role in ATM/CHK2 mediated DSB repair, it will be interesting to investigate the FOXO-dependent mechanisms controlling the establishment of the replication stress checkpoint (38-40).

Noteworthy, FOXOs have been suggested to play a role in inducing a G2 arrest, but these conclusions were based solely on DNA content analysis (41-44). As we do not observe mAG+ cells after FOXO activation, it's tempting to speculate that the previously reported FOXO-induced G2 arrest in fact represent cells that exited G2 and are in a G0/G1 state with 4n DNA. 
Combined, our results establish a novel role for FOXOs in the replication stress response. FOXOs cooperate with the ATR/CHK1 and p53/p21 response both by supporting the establishment of a checkpoint and simultaneously restricting the time in which cells are allowed to resolve the damaged DNA. Importantly, deregulation of this FOXO driven timer gives cells the opportunity to divide with higher levels of replication stress, potentially promoting cellular transformation.

\section{Materials \& Methods}

\section{Cell culture}

hTERT-immortalized Retinal Pigment Epithelial cells (RPE-1) were cultured in DMEMF12 (Lonza) containing 10\% FBS (Bodinco), 100U/ml penicillin and 100mg/ml streptomycin (Lonza). For doxycycline treatment $200 \mathrm{ng} / \mathrm{ml}$ Doxycycline (Sigma) was used for the duration of indicated times. RPE-1 cells in which a fluorescent tag was introduced in one allele of Cyclin B1 (RPE CCNB1 ${ }^{\text {YFP) }}$ ) have been described before (43).

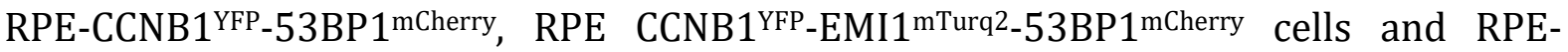
CCNB1 $^{\text {YFP-DHB }}{ }^{\text {mCherry }}$ cells were described before (11). Generation of RPE-FUCCI cells was previously described. Cells were treated with $0,2 \mu \mathrm{M}, 0,4 \mu \mathrm{M}$ aphidicolin (Sigma) for the indicated times. $0,2 \mu \mathrm{M}$ is used to induce replication stress from which cells can recover. $0,4 \mu \mathrm{M}$ induces a S/G2 arrest and was used to study APCCDH1 activation and replication stress checkpoint establishment.

\section{Constructs, lentiviral transduction and transfections}

Lentiviral cDNA expression vectors expressing FOX03.A3 were generated using Gateway cloning in the pINDUCER20 (Addgene \#44012) doxycycline inducible expression system (45). The lentiviral construct pCW-mTurqouise-EMI1 was generated as previously 
described (19). Transfecting third generation packaging vectors using Poly-ethylenimine into HEK293T cells generated lentiviral particles. Transfection of pTON-BIOPS-FlagEGFP-FOX01/FOXO3 and pCDNA-HA-E2F1 in HEK293T cells was performed using ExtremeGene 9 (Roche). Transfection of siRNA smartpools targeting EMI1, FZR1/CDH1 and E2F1 was performed using lipofectamine (Life Technologies).

CRISPR/Cas9 mediated knockouts we generated as previously described in (46) Briefly, pX330 was transfected in for both TP53 and CDKN1A knockout generation. Subsequently, cells were treated with $5 \mu$ M Nutlin-3a for 7 days to select out TP53 and CDKN1A deficient cells.

\section{Immunoblotting \& antibodies}

For western blot cells are lysed, protein concentration is measured (Bradford, BioRad) and finally dissolved in sample buffer containing $0.2 \%$ SDS, $10 \%$ glycerol, $0.2 \% \beta$ mercapto-ethanol, 60mM Tris pH6.8. Equal protein concentrations were loaded and proteins were detected using 6-15\% SDS-PAGE gels and subsequent western-blot analysis with primary antibodies recognizing FOXO1 (CS2880), FOXO3 (NB100614), p27 (BD-610241), p21 (BD556430), p53 (SC-126), EMI1 (ThermoFisher 3D2D6), E2F1 (CST3742), RB-S807/811 (CST-9308), Cyclin A (AB-16726), Cyclin E (SC-198), Cyclin D (ab134175), Cyclin B (SC-752), CDH1/FZR1 (MS-1116-P1), SKP2 (CST-2652S), CHK1 (SC8408), phospho-CHK1 (CD2348), CHK2 (SC9064), phospho-CHK2 (CS2261), FANCD2 (ab108928), 53BP1 (NB100-304), yH2AX (MP 05-636), HA (home made), GFP (home made), used 1:2000. Primary antibodies were detected by secondary HRP conjugated antibodies targeting mouse, rabbit, and rat IgG and visualized using chemiluminescence (Biorad) and an ImageQuant LAS 4000 scanner (GE Healthcare). For immunoprecipitation cells transfected with HA-E2F1, GFP-FOX01, GFP-FOXO3 were 
lysed in 50mM Tris pH7.4, 150mM NaCl, 1\% Triton-X100. For immunoprecipitation Chromotec GFP beads were incubated with cell lysate for 2 hours at $4^{\circ} \mathrm{C}$, subsequently washed with lysis buffer and boiled in sample buffer. Chromatin-IP was performed as previously described using rabbit anti E2F1 (C-20, SC193) and Rabbit IgG (SC2027) (34)

\section{Immunofluorescence}

Cells were grown on glass coverslips, fixed using 4\% paraformaldehyde and blocked with PBS containing $2 \%$ bovine serum albumin (BSA) (Invitrogen) and $0.1 \%$ normal goat serum (Invitrogen). Cells were incubated with indicated antibodies (1:500), secondary Alexa488/561 conjugated antibodies and Hoechst (Sigma). Slides were imaged on a Zeiss LSM710 confocal microscope. Quantification of FOXO localization and 53BP1, FANCD2 and $\gamma \mathrm{H} 2 \mathrm{AX}$ foci was performed with a custom written Image script that was described previously (19),

\section{RT-qPCR}

mRNA was isolated from live cells using the Qiagen RNeasy kit (Qiagen) and cDNA synthesis was performed using the iScript cDNA synthesis kit (BioRad). Real-time PCR was performed using Fast Start Universal SYBR Green Master (ROX) mix (Roche) in the CFX Connect Real-time PCR detection system (BioRad). Target genes were amplified using specific primer pairs (Supplemental table 1) and specificity was confirmed by analysis of the melting curves. Target gene expression levels were normalized to GAPDH and HNRNPA1 levels. 


\section{Flow cytometry}

For DNA content profiling and sorting, live cells were incubated with $10 \mu \mathrm{g} / \mathrm{ml}$ Hoechst33342 for $30 \mathrm{~min}$. at $37^{\circ} \mathrm{C}$. After incubation cells were trypsinized and transferred to normal culture medium before measuring. mKO-hCDT1, mAG-hGeminin and Hoechst33342 intensity was measured using a BD LSR Fortessa Flow cytometer or BD Aria II FACS (BD bioscience).

\section{Live cell imaging and tracking}

20.000 RPE cells were cultured in Lab-Tek II 8-well imaging chambers. Prior to imaging normal culture medium is replaced with Leibovitz medium (Lonza) containing 10\% FCS (Lonza),2 mM L-Glutamin, 100U/ml penicillin and 100 $\mu \mathrm{g} / \mathrm{ml}$ streptomycin (Lonza). Cells were treated with $0.4 \mu \mathrm{M}$ aphidicolin and/or doxycycline (Sigma) at indicated timepoints before imaging. For UV irradiation, medium was aspirated and cells were rinsed with PBS before exposure to global UV irradiation by TUV lamp. After irradiation Leibovitz's L-15 (Gibco) CO2-independent medium, supplemented with ultra-glutamine, penicillin/streptomycin and 10\% fetal calf serum was added to start live-cell imaging as described above. For all experiments where phenotypic outcome was quantified at least 50 cells per condition in each independent biological replicate were scored, $n \geq 50$, unless otherwise stated in figure legends. Imaging was performed on Zeiss Cell observer RealTime imaging and DeltaVision Elite (applied precision) microscopes for 48 hours at $37^{\circ} \mathrm{C}$. Cell tracking and quantification was performed using ImageJ. Cells expressing mAGhGeminin at the moment of doxycycline addition and cell starting to express mAGhGeminin within 3 hours after doxycycline addition were considered S/G2 in the analysis. Relative CDK2 activity and cyclin B1 ${ }^{\mathrm{YFP}}$ intensity were measured in individual G2 cells that degraded Cyclin B1 after progression through S phase in presence of $0,4 \mu \mathrm{M}$ 
aphidicolin. Cells were in silico alligned at the onset of cyclin B1 degradation and cyclin B1 ${ }^{\text {YFP }}$ values were normalized to the max cyclin B1 level.

\section{mRNA sequencing \& Analysis}

RPE-1 hTERT cells were synchronized with a double-thymidine block and released in the absence (DMSO) and presence of aphidicolin (DRS) and cultured for 7 hours. Subsequently, total RNA from cultured cells was extracted using TRIzol reagent (Invitrogen). Strand-specific libraries were generated using the TruSeq PolyA Stranded mRNA sample preparation kit (illumina). In brief, polyadenylated RNA was purified using oligo-dT beads. Following purification, the RNA was fragmented, random-primed and reserve transcribed using SuperScript II Reverse Transcriptase (Invitrogen). The generated cDNA was $3^{\prime}$ end-adenylated and ligated to Illumina Paired-end sequencing adapters and amplified by PCR using HiSeq SR Cluster Kit v4 cBot (Illumina). Libraries were analyzed on a 2100 Bioanalyzer (Agilent) and subsequently sequenced on a HiSeq2000 (Illumina). We performed RNaseq alignment using TopHat 2.1.1 (Supplemental table 2) (47). Differentially expressed genes were called with DEseq2 (48) with an adjusted $\mathrm{p}$ value threshold of $<0,005$. Transcription factor binding site analysis was performed using oPOSSUM (49) to determine enrichment for transcription factors by Z-score and Fisher exact score over the whole genome compared to our upregulated genes (Supplemental table 2).

\section{Author contributions:}

MH, FF, LK, LS, JB, BB performed experiments. MH, FF, LK, JB wrote the manuscript. NN, MR supported cell sorting. TD, RM, BB financially supported this study and provided critical feedback. 


\section{Acknowledgements}

This study is financially supported by CancerGenomicsCenter.nl, the Oncode institute and the research grants UU2014-6902, UU2009-4490 (to TD) and NKI2017-6787 (to RM) from the Dutch Cancer Society (KWF kankerbestrijding).

\section{Conflict of interest}

The authors declare no conflict of interest.

\section{References}

1. Zeman MK, Cimprich KA. Causes and consequences of replication stress. Nat Cell Biol. 2014 Jan 24;16(1):2-9.

2. Arora M, Moser J, Phadke H, Basha AA, Spencer SL. Endogenous Replication Stress in Mother Cells Leads to Quiescence of Daughter Cells. Cell Rep. 2017 May 16;19(7):1351-64.

3. Harrigan JA, Belotserkovskaya R, Coates J, Dimitrova DS, Polo SE, Bradshaw CR, et al. Replication stress induces 53BP1-containing OPT domains in G1 cells. J Cell Biol. 2011 Apr 4;193(1):97-108.

4. Lukas C, Savic V, Bekker-Jensen S, Doil C, Neumann B, Pedersen RS, et al. 53BP1 nuclear bodies form around DNA lesions generated by mitotic transmission of chromosomes under replication stress. Nat Cell Biol. 2011 Mar 13;13(3):243-53.

5. Koundrioukoff S, Carignon S, Técher H, Letessier A, Brison O, Debatisse M. Stepwise Activation of the ATR Signaling Pathway upon Increasing Replication Stress Impacts Fragile Site Integrity. PLoS Genet. 2013;9(7):e1003643.

6. Marusyk A, Wheeler LJ, Mathews CK, DeGregori J. p53 Mediates Senescence-Like Arrest Induced by Chronic Replicational Stress. Mol Cell Biol. 2007 
Aug;27(15):5336-51.

7. Glover TW, Berger C, Coyle J, Echo B. DNA polymerase $\alpha$ inhibition by aphidicolin induces gaps and breaks at common fragile sites in human chromosomes. Hum Genet. 1984;67(2):136-42.

8. Mishmar D, Rahat A, Scherer SW, Nyakatura G, Hinzmann B, Kohwi Y, et al. Molecular characterization of a common fragile site (FRA7H) on human chromosome 7 by the cloning of a simian virus 40 integration site. Proc Natl Acad Sci. 1998 Jul 7;95(14):8141-6.

9. Feringa FM, Krenning L, Koch A, van den Berg J, van den Broek B, Jalink K, et al. Hypersensitivity to DNA damage in antephase as a safeguard for genome stability. Nat Commun. 2016 Aug;7:12618.

10. Krenning L, Feringa FM, Shaltiel IA, vandenBerg J, Medema RH. Transient activation of p53 in G2 phase is sufficient to induce senescence. Mol Cell. 2014 Jul;55(1):59-72.

11. Wiebusch L, Hagemeier C. P53-and p21-dependent premature APC/C-Cdh1 activation in G2 is part of the long-term response to genotoxic stress. Oncogene. 2010 Jun 12;29(24):3477-89.

12. Sudo T, Ota Y, Kotani S, Nakao M, Takami Y, Takeda S, et al. Activation of Cdh1dependent APC is required for G1 cell cycle arrest and DNA damage-induced G2 checkpoint in vertebrate cells. EMBO J. 2001 Nov;20(22):6499-508.

13. Zachariae W, Schwab M, Nasmyth K, Seufert W. Control of cyclin ubiquitination by CDK-regulated binding of Hct1 to the anaphase promoting complex. Science (80). 1998 Nov 27;282(5394):1721-4.

14. Kramer ER, Scheuringer N, Podtelejnikov A V., Mann M, Peters JM. Mitotic regulation of the APC activator proteins CDC20 and CDH1. Mol Biol Cell. 2000 
May;11(5):1555-69.

15. Spencer SL, Cappell SD, Tsai FC, Overton KW, Wang CL, Meyer T. XThe proliferation-quiescence decision is controlled by a bifurcation in CDK2 activity at mitotic exit. Cell. 2013 Oct 10;155(2):369.

16. Sudo T, Ota Y, Kotani S, Nakao M, Takami Y, Takeda S, et al. Activation of Cdh1dependent APC is required for G1 cell cycle arrest and DNA damage-induced G2 checkpoint in vertebrate cells. EMBO J. 2001 Nov 15;20(22):6499-508.

17. Lee J, Kim JA, Barbier V, Fotedar A, Fotedar R. DNA damage triggers p21 WAF1dependent emil down-regulation that maintains G2 arrest. Mol Biol Cell. 2009 Apr;20(7):1891-902.

18. Müllers E, Cascales HS, Jaiswal H, Saurin AT, Lindqvist A. Nuclear translocation of Cyclin B1 marks the restriction point for terminal cell cycle exit in G2 phase. Cell Cycle. 2014;13(17):2733-43.

19. Feringa FM, Krenning L, Koch A, Van Den Berg J, Van Den Broek B, Jalink K, et al. Hypersensitivity to DNA damage in antephase as a safeguard for genome stability. Nat Commun. 2016 Nov 26;7(1):12618.

20. Reimann JDR, Freed E, Hsu JY, Kramer ER, Peters JM, Jackson PK. Emi1 is a mitotic regulator that interacts with Cdc20 and inhibits the anaphase promoting complex. Cell. 2001 Jun 1;105(5):645-55.

21. Hsu JY, Reimann JDR, Sørensen CS, Lukas J, Jackson PK. E2F-dependent accumulation of hEmi1 regulates S phase entry by inhibiting APCCdh1. Nat Cell Biol. 2002 May 22;4(5):358-66.

22. Miller JJ, Summers MK, Hansen D V., Nachury M V., Lehman NL, Loktev A, et al. Emi1 stably binds and inhibits the anaphase-promoting complex/cyclosome as a pseudosubstrate inhibitor. Genes Dev. 2006 Sep 1;20(17):2410-20. 
23. Di Fiore B, Pines J. Emi1 is needed to couple DNA replication with mitosis but does not regulate activation of the mitotic APC/C. J Cell Biol. 2007 May 7;177(3):425-37.

24. Saldivar JC, Cortez D, Cimprich KA. The essential kinase ATR: Ensuring faithful duplication of a challenging genome. Nat Rev Mol Cell Biol. 2017;18(10):622-36.

25. Rashi-Elkeles S, Warnatz H-J, Elkon R, Kupershtein A, Chobod Y, Paz A, et al. Parallel profiling of the transcriptome, cistrome, and epigenome in the cellular response to ionizing radiation. Sci Signal. 2014 May 13;7(325):rs3.

26. Brown AK, Webb AE. Regulation of FOXO Factors in Mammalian Cells. In: Current topics in developmental biology. 2018. p. 165-92.

27. Hornsveld M, Dansen TB, Derksen PW, Burgering BMT. Re-evaluating the role of FOXOs in cancer. Semin Cancer Biol. 2018 Jun;50:90-100.

28. Charitou P, Rodriguez-Colman M, Gerrits J, Triest M, Groot Koerkamp M, Hornsveld M, et al. FOXO s support the metabolic requirements of normal and tumor cells by promoting IDH 1 expression. EMBO Rep. 2015 Apr 3;16(4):45666.

29. Medema RH, Kops GJPL, Bos JL, Burgering BMT. AFX-like Forkhead transcription factors mediate cell-cycle regulation by Ras and PKB through p27kip1. Nature. 2000 Apr 13;404(6779):782-7.

30. Hornsveld M, Smits LMM, Meerlo M, van Amersfoort M, Groot Koerkamp MJA, van Leenen D, et al. FOXO Transcription Factors Both Suppress and Support Breast Cancer Progression. Cancer Res. 2018 May 1;78(9):2356-69.

31. de Keizer PLJ, Packer LM, Szypowska AA, Riedl-Polderman PE, van den Broek NJF, de Bruin A, et al. Activation of forkhead box 0 transcription factors by oncogenic BRAF promotes p21cip1-dependent senescence. Cancer Res. 2010 Nov 
$1 ; 70(21): 8526-36$.

32. Shats I, Gatza ML, Liu B, Angus SP, You L, Nevins JR. FOXO transcription factors control E2F1 transcriptional specificity and apoptotic function. Cancer Res. 2013 Oct 1;73(19):6056-67.

33. Margottin-Goguet F, Hsu JY, Loktev A, Hsieh HM, Reimann JDR, Jackson PK. Prophase destruction of Emi1 by the SCF $\beta \operatorname{TrCP} /$ Slimb ubiquitin ligase activates the anaphase promoting complex to allow progression beyond prometaphase. Dev Cell. 2003 Jun 1;4(6):813-26.

34. Eijkelenboom A, Mokry M, de Wit E, Smits LM, Polderman PE, van Triest MH, et al. Genome-wide analysis of FOXO3 mediated transcription regulation through RNA polymerase II profiling. Mol Syst Biol. 2013 Jan 22;9(1):638.

35. Eijkelenboom A, Mokry M, Smits LM, Nieuwenhuis EE, Burgering BMT. FOXO3 Selectively Amplifies Enhancer Activity to Establish Target Gene Regulation. Cell Rep. 2013 Dec 26;5(6):1664-78.

36. Nakayama K, Nagahama H, Minamishima YA, Matsumoto M, Nakamichi I, Kitagawa K, et al. Targeted disruption of Skp2 results in accumulation of cyclin E and p27Kip1, polyploidy and centrosome overduplication. EMBO J. 2000 May 2;19(9):2069-81.

37. Nakayama K, Nagahama H, Minamishima YA, Miyake S, Ishida N, Hatakeyama S, et al. Skp2-mediated degradation of p27 regulates progression into mitosis. Dev Cell. 2004 May;6(5):661-72.

38. Chung YM, Park S-H, Tsai W-B, Wang S-Y, Ikeda M-A, Berek JS, et al. FOXO3 signalling links ATM to the p53 apoptotic pathway following DNA damage. Nat Commun. 2012 Jan 14;3(1):1000.

39. Tsai W-B, Chung YM, Takahashi Y, Xu Z, Hu MC-T. Functional interaction between 
FOX03a and ATM regulates DNA damage response. Nat Cell Biol. 2008 Apr $16 ; 10(4): 460-7$.

40. Adamowicz M, Vermezovic J, d'Adda di Fagagna F. NOTCH1 Inhibits Activation of ATM by Impairing the Formation of an ATM-FOX03a-KAT5/Tip60 Complex. Cell Rep. 2016 Aug 23;16(8):2068-76.

41. Tran H, Brunet A, Grenier JM, Datta SR, Fornace AJ, DiStefano PS, et al. DNA Repair Pathway Stimulated by the Forkhead Transcription Factor FOXO3a Through the Gadd45 Protein. Science (80- ). 2002 Apr 19;296(5567):530-4.

42. Furukawa-Hibi Y, Yoshida-Araki K, Ohta T, Ikeda K, Motoyama N. FOXO Forkhead Transcription Factors Induce G 2 -M Checkpoint in Response to Oxidative Stress. J Biol Chem. 2002 Jul 26;277(30):26729-32.

43. Schmidt M, Fernandez de Mattos S, van der Horst A, Klompmaker R, Kops GJPL, Lam EW-F, et al. Cell cycle inhibition by FoxO forkhead transcription factors involves downregulation of cyclin D. Mol Cell Biol. 2002 Nov;22(22):7842-52.

44. Alvarez B, Martínez-A C, Burgering BM, Carrera AC. Forkhead transcription factors contribute to execution of the mitotic programme in mammals. Nature. 2001 Oct 18;413(6857):744-7.

45. Hornsveld M, Smits LMM, Meerlo M, Van Amersfoort M, Groot Koerkamp MJA, van Leenen D, et al. FOXO transcription factors both suppress and support breast cancer progression. Cancer Res. 2018;78(9).

46. Blomen VA, Majek P, Jae LT, Bigenzahn JW, Nieuwenhuis J, Staring J, et al. Gene essentiality and synthetic lethality in haploid human cells. Science (80- ). 2015 Nov 27;350(6264):1092-6.

47. Kim D, Pertea G, Trapnell C, Pimentel H, Kelley R, Salzberg SL. TopHat2: accurate alignment of transcriptomes in the presence of insertions, deletions and gene 
fusions. Genome Biol. 2013 Apr 25;14(4):R36.

48. Love MI, Huber W, Anders S. Moderated estimation of fold change and dispersion for RNA-seq data with DESeq2. Genome Biol. 2014 Dec 5;15(12):550.

49. Ho Sui SJ, Fulton DL, Arenillas DJ, Kwon AT, Wasserman WW. oPOSSUM: integrated tools for analysis of regulatory motif over-representation. Nucleic Acids Res. 2007 Jul;35(suppl_2):W245-52.

\section{Figure 1: Replication stress leads to APC ${ }^{\mathrm{CDH} 1}$ activation in G2 phase.}

A Representative image from time-lapse movies of RPE CCNB ${ }^{\text {YFP }}-53 B P 1^{\text {mCherry }}$ cells treated with $0,4 \mu \mathrm{M}$ aphidicolin. B Mitotic entry of G2 cells (Cyclin B1 positive at $\mathrm{t}=0$ ) or S phase cells (Cyclin B1 positive at $\mathrm{t}=15$ ) in the presence or absence of $0,4 \mu \mathrm{M}$ aphidicolin. Average of three independent experiments + sem. C Percentage of cells from figure B that degrade Cyclin B1 in the presence or absence of $0,4 \mu \mathrm{M}$ aphidicolin. Average of three independent experiments + sem. D Quantification of 53BP1mCherry foci in G2 cells before onset of Cyclin B1 degradation or mitotic entry. Dots represent individual cells pooled from two independent experiments. Mean + sd. ${ }^{* * * *} \mathrm{P}<0.0001$ (Welch's corrected unpaired t-test). E Percentage of G2 cells that degrade cyclin following aphidicolin treatment in presence of FZR1 knock-down. F Representative flow cytometric analysis of hoechst stained RPE-FUCCI cells after $0,4 \mu \mathrm{M}$ aphidicolin treatment for 24,48 and 72 hours. Red: CDT1-mKO+ (G1-phase), green: Geminin-mAG+ (S/G2/M-phase). G Flow cytometry quantification of cell cycle distribution of $4 \mathrm{n}$ cells in hoechst stained RPEFUCCI cells knockout for p53 ( $\Delta$ p53) and $(\Delta$ p21) (n=3). Red: CDT1-mKO+ (G1-phase), green: Geminin-mAG+ (S/G2/M-phase). H Relative CDK2 activity and cyclin B1YFP intensity in individual G2. Lines represent average of 16 cells pooled from two independent experiments $+95 \%$ CI. I Cumulative mitotic entry of G2 cells that progressed 
through S phase after treatment with $0,2 \mu \mathrm{M}$ aphidicolin in presence or absence of EMI1 overexpression or ATR inhibitor. Average of three independent experiments + sem.

Figure 2: FOXO3 is activated by replication stress and induces cell cycle exit from

\section{G2 phase.}

A Volcano plot showing differentially expressed genes comparing aphidicolin-treated RPE-1 cells (7hrs) vs untreated RPE-1 (non DE = grey, $\mathrm{DE}=$ Black $((\mathrm{p}<0.005 \mid-$ $0.5<\log 2 \mathrm{FC}>0.5)$ ), DE genes with at least 1 FOXO Transcription factor binding sites $($ TFBS $)=$ red $)$ after RNA-sequencing of aphidicolin treated RPE cells. B Venn diagram illustrating the overrepresentation of FOXO TFBS in promoters amongst upregulated genes (116) to the total all promoters (24752). C Immunofluorescence image of FOXO3 (Grey) expression and localization in RPE-FUCCI cells. Red: CDT1-mKO+ (G1-phase), green: Geminin-mAG+ (S/G2/M-phase). D Violin plot of average nuclear intensity of F0X03 in the absence/presence of $0,4 \mu \mathrm{M}$ aphidicolin or $5 \mu \mathrm{M}$ ATR inhibitor VE-821. Solid line $=$ median, dotted line $=$ quartile. Welch's corrected unpaired t-test $\mathrm{P}<0.05=*$, $\mathrm{p}<0.05=* *$. E Representative fluorescence time lapse images of RPE-FUCCI-iFOXO3.A3 cells that shows cell cycle exit 18h after FOX03.A3 induction. A geminin-mAG (green) positive cell becomes CDT1-mKO (red) positive without progressing through mitosis. FG Flow cytometric analysis example and quantification of hoechst stained RPE-FUCCI cells after 30h FOX03.A3 expression (FOXO ON), 30h 0,4 $\mathrm{M}$ aphidicolin, combined treatment and FOX03.A3 expression in cells pretreated with aphidicolin for $24 \mathrm{~h}$. Red: CDT1-mKO+ (G1-phase), green: Geminin-mAG+ (S/G2/M-phase). H Flow cytometry quantification of $4 \mathrm{n}$ CDT1-mKO+ cells after FOXO activation in p53 $(\Delta \mathrm{p} 53)$ and p21 $(\Delta \mathrm{p} 21)$ knockout RPE-FUCCI cells $(\mathrm{n}=3)$. 


\section{Figure 3: FOXOs support replication stress response and cooperate with p53/p21}

\section{to induce cell cycle exit.}

A Westernblot for p21, p53, F0X01and F0X03 levels in RPE-FUCCI, p21 ( $\Delta$ p21) and p53 $(\Delta \mathrm{p} 53)$ knockout cells before and after $0,4 \mu \mathrm{M}$ aphidicolin treatment in combination with shFOXOs. Nonspecific background staining was used as loading. B Representative flow cytometric analysis of hoechst stained RPE-FUCCI, p21 ( $\Delta$ p21) and p53 ( $\Delta$ p53) knockout cells before and after $0,4 \mu \mathrm{M}$ aphidicolin treatment in combination with shFOXOs. Red: CDT1-mKO+ (G1-phase), green: Geminin-mAG+ (S/G2/M-phase). C Flow cytometric quantification of $4 \mathrm{n}$ CDT1-mKO+ cells after $0,4 \mu \mathrm{M}$ aphidicolin treatment in combination with shF0X0s of p53 $(\Delta \mathrm{p} 53)$ and p21 $(\Delta \mathrm{p} 21)$ knockout RPE-FUCCI cells $(\mathrm{n}=3)$. D Westernblot for phospho-CHK1, CHK1, phospho-CHK2, CHK2 and FOXO3 expression levels after $0,4 \mu \mathrm{M}$ aphidicolin treatment in combination with Hydroxyurea (HU) or Neocarcinostatin treatment (NCS). Nonspecific background staining was used as loading. E Violin plot representing immunofluorescence quantification of the amount of $\gamma \mathrm{H} 2 \mathrm{AX}$ foci in control $(n=821)$, Aph $(n=88)$, shFOXOs $(n=191)$ and combined Aph + shFOXOs $(\mathrm{n}=71)$ treated cells. Median $=$ solid line, quartiles $=$ dashed line. Welch's corrected unpaired t-test $\mathrm{P}<0.00005=* * * *$. F Violin plot representing immunofluorescence quantification of the amount of 53BP1 foci in control ( $n=1553)$, Aph ( $n=1059)$, shFOXOs $(\mathrm{n}=801)$ and combined Aph + shFOXOs treated cells $(\mathrm{n}=1639)$. Median $=$ solid line, quartiles $=$ dashed line. Welch's corrected unpaired t-test $\mathrm{P}<0.00005={ }^{* * * *}$. G Violin plot representing immunofluorescence quantification of the amount of FANCD2 foci in control $(n=821)$, Aph $(n=273)$, shFOXOs $(n=116)$ and combined Aph + shFOXOs $(n=655)$ treated cells. Median $=$ solid line, quartiles $=$ dashed line . Welch's corrected unpaired t-test $\mathrm{P}<0.0005=* * *, \mathrm{p}<0.00005=* * * * . \mathrm{H}$ Westernblot for mono-ubiquitinated FANCD2 and 
F0X03 expression levels after $0,4 \mu \mathrm{M}$ aphidicolin treatment for 2 and 24 hours in combination with FOXO knockdown. Nonspecific background staining was used as loading control. I Dose response curve of cell viability after treatment with aphidicolin for 7 days in RPE-FUCCI cells with expressing shLuc or shFOXOs. Bonferonni Muiltiple testing corrected ANOVA p-value $<0.05=*, \mathrm{p}<0.005=* * *,<0.0005=* * * *$.

\section{Figure 4: FOXOs activate APCCDH1 in G2 by downregulating EMI1.}

A Westernblot for EMI1 and FOXO3 levels after 8, 10, 12, 14, 16, 18, 20, 22 and 24h FOXO activation in asynchronous RPE-FUCCI cells. Nonspecific background staining was used as loading. B Westernblot for EMI1, phospho-RB, FOX03, Cyclin A and Cyclin B in CDT1mKO+ and Geminin-mAG+ sorted cells after 8 and 16h FOXO activation. Nonspecific background staining was used as loading. C qPCR analysis of EMI1 expression in CDT1mKO+ and Geminin-mAG+ sorted cells after 16h FOXO activation. D Flow cytometric analysis of hoechst stained RPE-FUCCI-iFOX03.A3 before and after 30h F0X03.A3 expression in combination with siEMI1. \% 4n n=3. Red: CDT1-mKO+, green: GemininmAG+. E Westernblot for EMI1, mTurq2-EMI1 and F0X03.A3 expression in RPE-FUCCIiFOX03.A3+EMI1 cells. Nonspecific background staining was used as loading control. F Cumulative mitotic entry of G2 cells that progressed through S phase after FOXO3.A3 and EMI1 overexpression. Average of three independent experiments + sem. G Westernblot for EMI1 and FOX03.A3 expression in MAG+ sorted RPE-FUCCI-shFOXOs cells after 24 and $48 \mathrm{~h} 0,4 \mu \mathrm{M}$ aphidicolin treatment. Nonspecific background staining was used as loading control. H qPCR analysis of EMI1 expression in mAG+ sorted cells after 24 and 48h FOXO activation. I Average \% input of qPCR of genomic E2F1 binding sites in 2 promoter regions of EMI1 (EMI1a \& Emi1b), CCNE2 and CCNA2 after E2F1 chromatin immunoprecipitation in the presence/absence of F0X03.A3 expression $(n=3)$. J Flow 
cytometric analysis of hoechst stained RPE-FUCCI-iFOXO3.A3 before and after 30h FOXO activation in combination with siE2F1. \% 4n n=3 K Cumulative mitotic entry of G2 cells that progressed through S phase after FOX03.A3 overexpression in the precence or absence of siSCR and siE2F1. Average of three independent experiments + sem. $\mathbf{L}$ Westernblot for EMI1, Cyclin B, Cyclin A, Cyclin D, phospho-RB, p27, p21, FOXO3, CDH1 and SKP2 levels in 2n mKO+, 4n mAG+ 4n mkO+ sorted RPE-FUCCI-iFOXO3.A3 cells after 24 hours FOXO activation. Nonspecific background staining was used as loading. M Schematic illustrating that FOXOs reduce the levels of EMI1 in response to replication stress. When EMI1 levels reach critically low levels, the APC/C ${ }^{\mathrm{CDH} 1}$ (red line) activates, SCFSKP2 (green line) subsequently is inactivated and cell cycle exit from G2 is triggered.

\section{Supplementary figure 1.}

Quantification of 53BP1mCherry foci in G1 after mitotis in the precense of $0,2 \mathrm{mM}$ aphidicolin and/or ATR inhibitor for $>15 \mathrm{~h}$. Dots represent individual cells pooled from two independent experiments. Mean $+\mathrm{sd} .{ }^{* *} \mathrm{P}<0.001$ (unpaired t-test). B Percentage of G2 cells that retain cytoplasmic cyclin B1 expression during the course of the exp (16 h) following aphidicolin treatment in presence of indicated knock-down. C Percent of G2 cells that degrade Cyclin B1 after combinational treatment of $0,4 \mu \mathrm{M}$ aphidicolin with mTurq2-EMI1 expression (dox) or 5 $\mu$ M ATR inhibitor VE-821.

\section{Supplemental figure 2 .}

A Immunofluorescence image of FOX01 (Grey) expression and localization in RPE-FUCCI cells $(\mathrm{CDT} 1(\mathrm{G} 1)=$ Red, Geminin $(\mathrm{S} / \mathrm{G} 2 / \mathrm{M})=$ green $)$ after Aph or $1 \mu \mathrm{M}$ AKT inhibitor treatment. B RT-qPCR analysis of FOXO1, FOXO3 and FOXO4 mRNA expression level changes in RPE-FUCCI-shFOXOs cells during 48 hours of $0,4 \mu \mathrm{M}$ Aph treatment in the 
absence or presence of shFOXOs. C Westernblot for FOX03.A3 expression after 16h doxycycline (dox) treatment. Nonspecific background staining was used as loading.

\section{Supplemental Figure 3.}

A\&B RT-qPCR analysis of P21 and P53 mRNA expression level changes in RPE-FUCCIiFOX03.A3 cells over the course of 24 hours doxycycline (dox) treatment. C Western blot analysis of p21, p53 and F0X03 protein levels in RPE-FUCCI-iFOXO3.A3 after doxycycline treatment for 24 hours. Equal concentrations of protein are loaded and aspecific background staining is used to visualize equal loading.

\section{Supplemental figure 4 .}

A Western blot analysis of FZR1/CDH1 protein levels in RPE-FUCCI-iFOX03.A3 after 72 hours of siSCR or siCDH1 with doxycycline treatment for 30 hours. Equal concentrations of protein are loaded and aspecific background staining is used to visualize loading. B Representative flow cytometric analysis of hoechst stained RPE-FUCCI-iFOXO3.A3 before and after 30h FOXO activation in combination with siCDH1. Red: CDT1-mKO+ (G1-phase), green: Geminin-mAG+ (S/G2/M-phase). C Flow cytometric quantification of 4n G1 cells after 30h FOXO activation in combination with siCDH1 (n=3). D RT-qPCR analysis of EMI1 expression in RPE-FUCCI-iFOX03.A3 cells over the course of 24 hours FOXO3.A3 expression. E Western blot analysis of EMI1 protein levels in RPE-FUCCI-iFOX03.A3 after 72 hours of siSCR or siEMI1 in combination with doxycycline treatment for 30 hours. Equal concentrations of protein are loaded and aspecific background staining is used to visualize loading. F Representative live imaging sequence of RPE-FUCCI-FOXO3.A3+EMI1 
cells visualizing the mitotic arrest observed after combined FOXO3 and EMI1 overexpression (dox). Red = mKO2 (G1 phase), Green is mAG. (S/G2/M phase)

\section{Supplemental figure 5 .}

A Westernblot for HA-E2F1, GFP-FOXO1 and GFP-FOXO3 protein levels after GFP immunoprecipitation in the presence/absence of $10 \mu \mathrm{M}$ AKT inhibitor VIII for 60 min. Nonspecific background staining was used as loading. B-D RT-qPCR analysis of E2F1, CCNE2, CCNA2 mRNA expression level changes in RPE-FUCCI-iFOXO3.A3 cells over the course of 24 hours doxycycline treatment (FOXO ON). E Western blot analysis of E2F1 protein levels in RPE-FUCCI-iFOX03.A3 after 72 hours of siSCR or siE2F1 in combination with doxycycline treatment for 8 hours. Equal concentrations of protein are loaded and aspecific background staining is used to visualize equal protein content. 


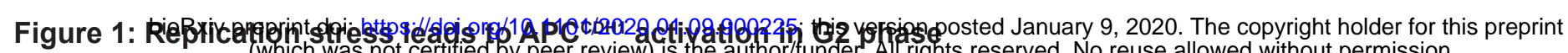
which was not certified by peer review) is the author/funder. Alf rights reserved. No reuse allowed without permission.

A
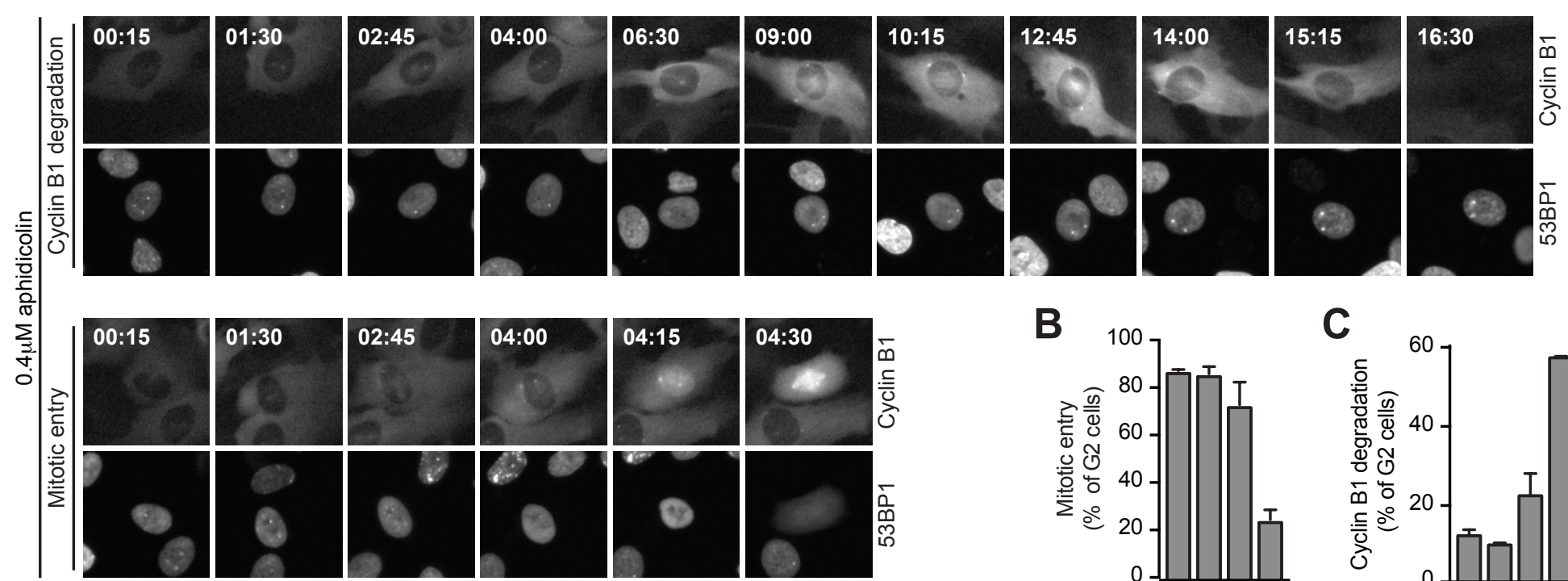

E

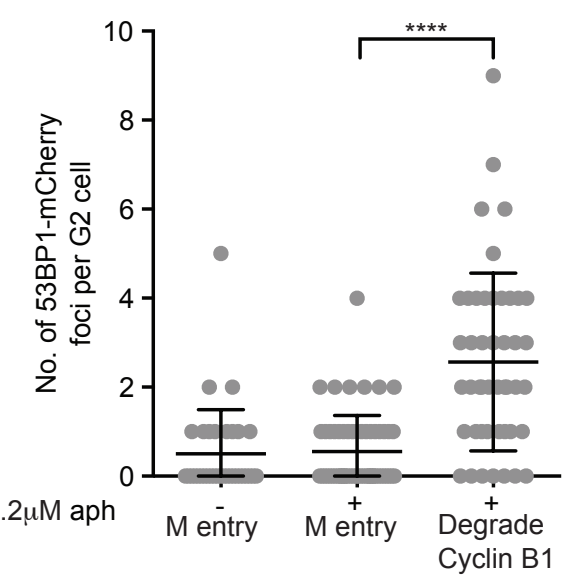

G

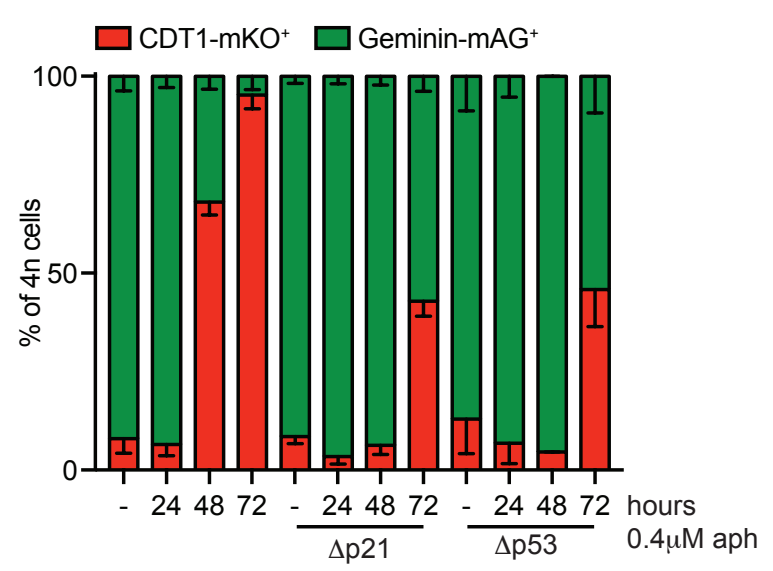

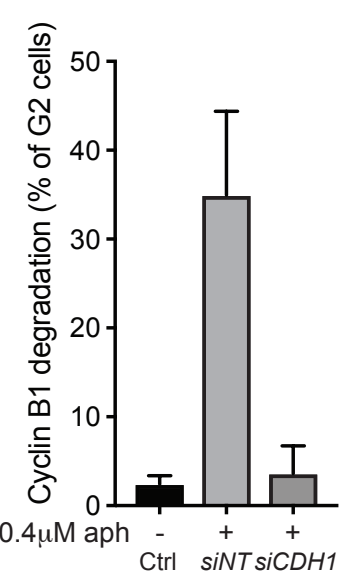

H

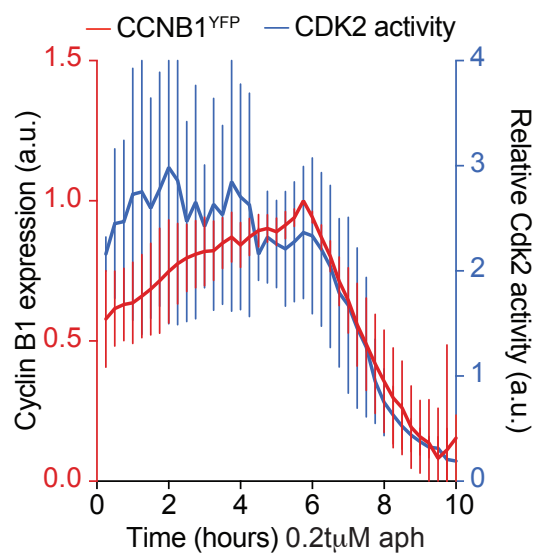

B

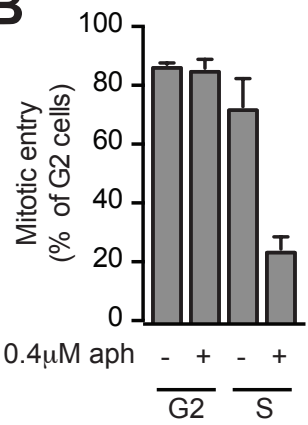

$\mathbf{F}$
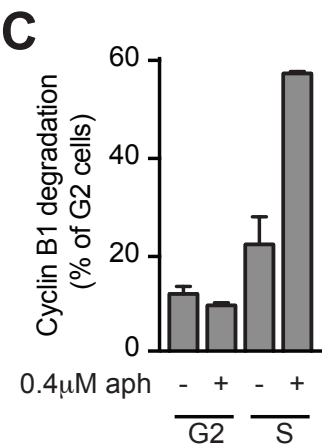

$0.4 \mu \mathrm{M}$ aph

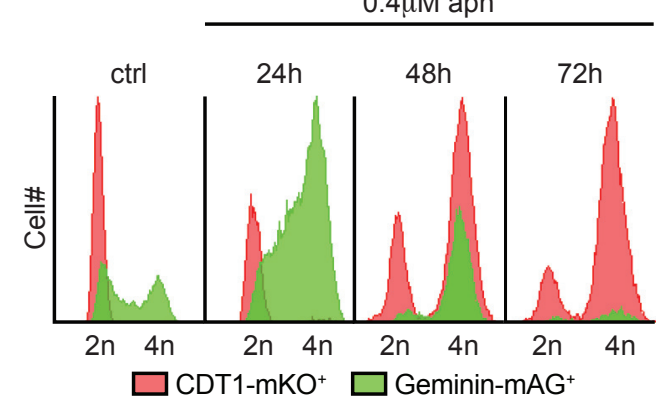

I

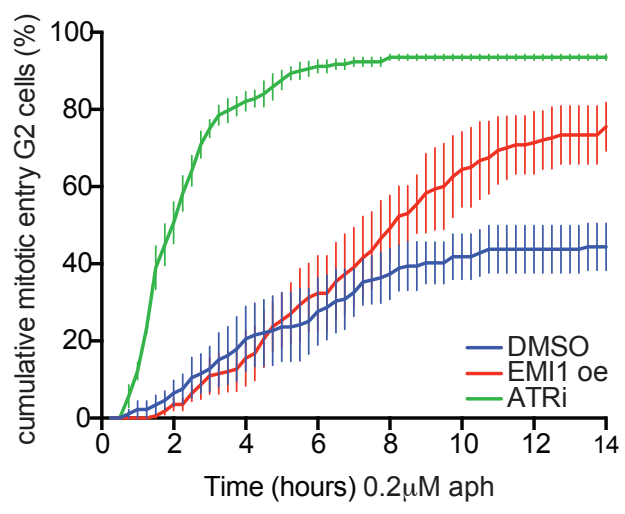


A

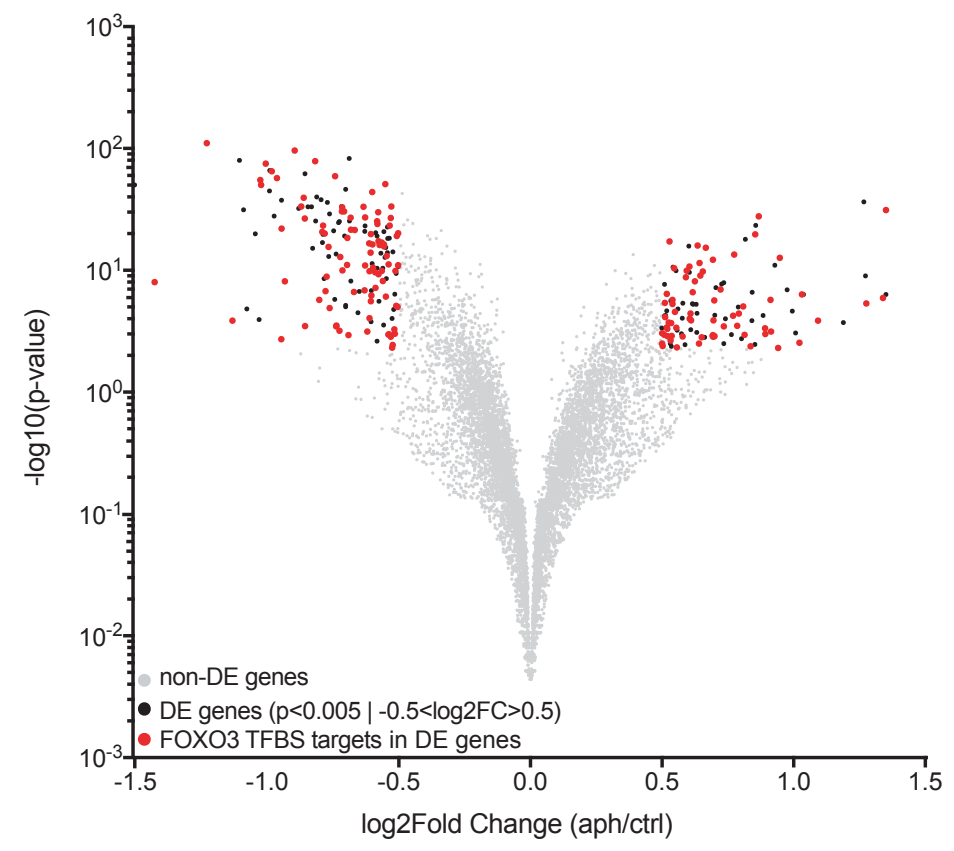

C

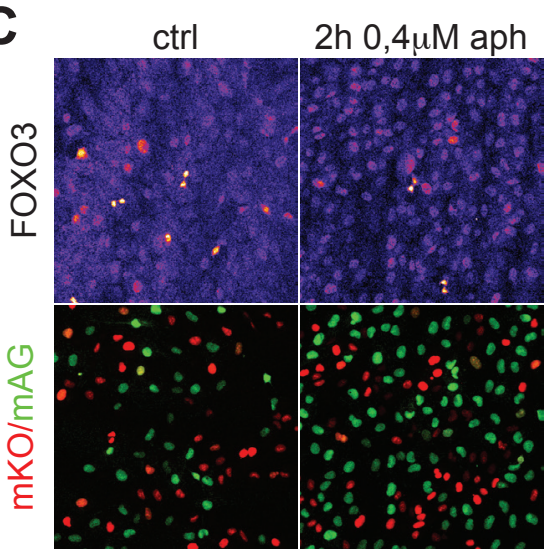

$\mathbf{F}$
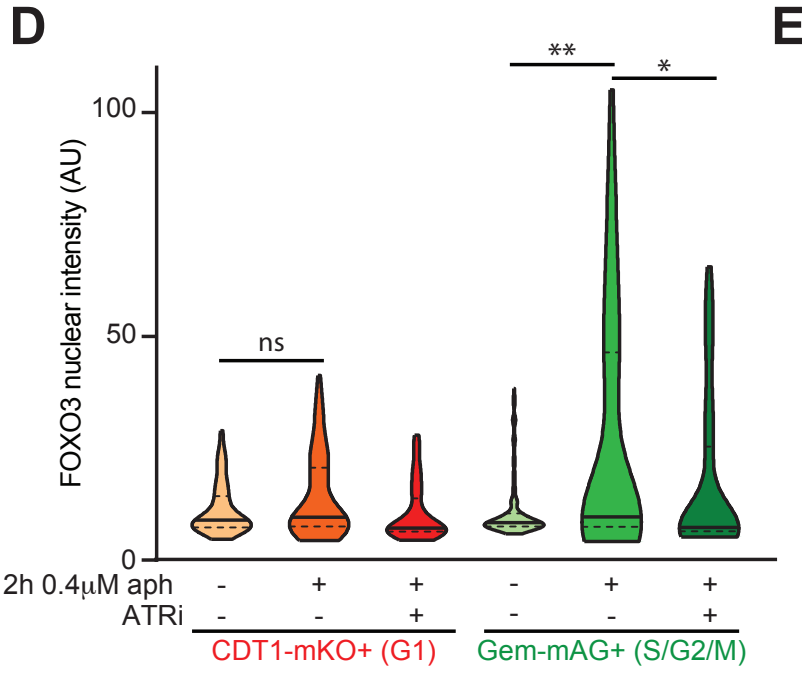

G

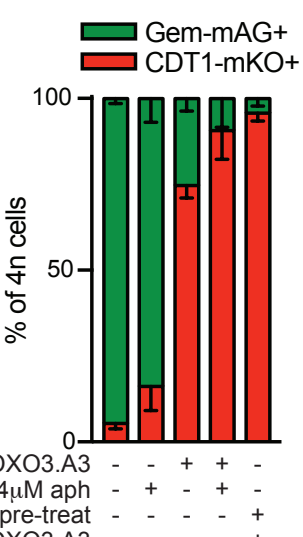

E
Upregulated genes

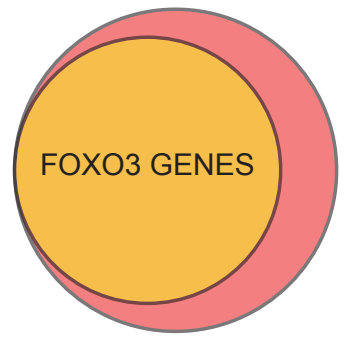

116
Z-score: 23.506

Fisher Score: 5.749

RPE-FUCCI-iFOXO3.A3 + dox at $\mathrm{t}=0$

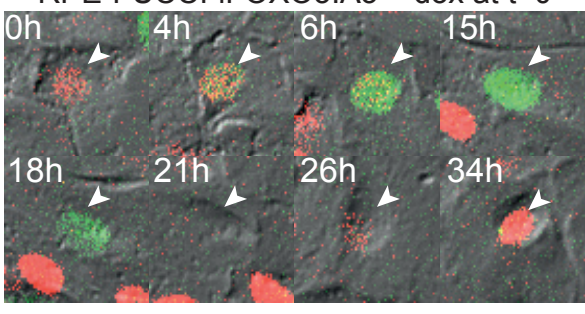

$\square$ CDT1-mKO $^{+} \square$ Geminin-mAG ${ }^{+}$

H

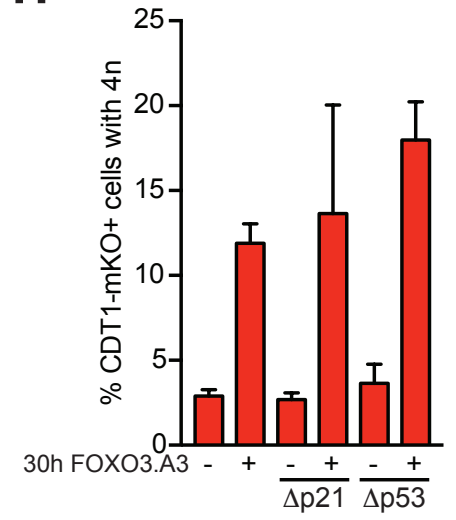




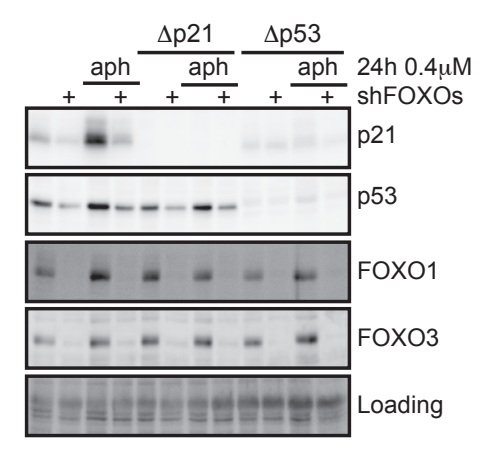

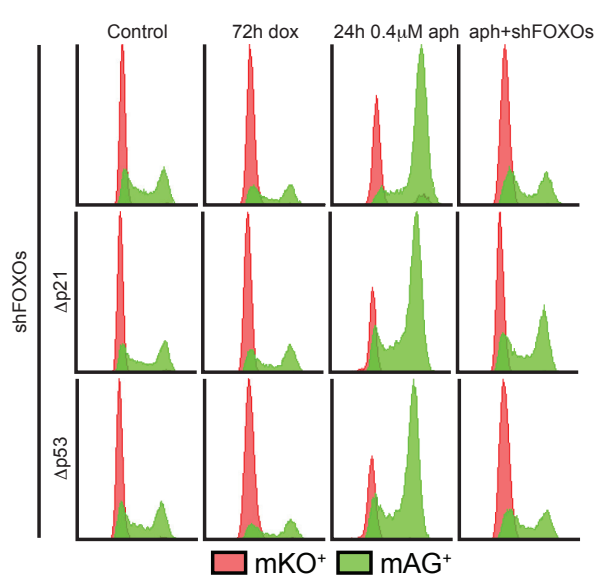

E

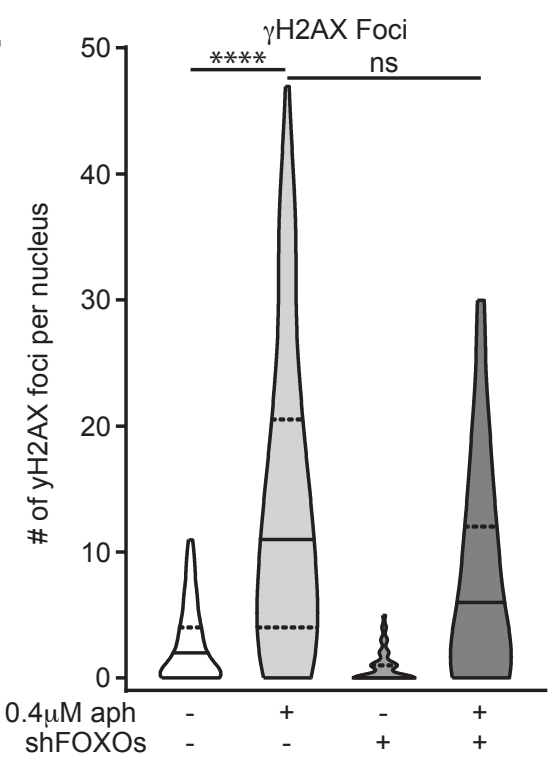

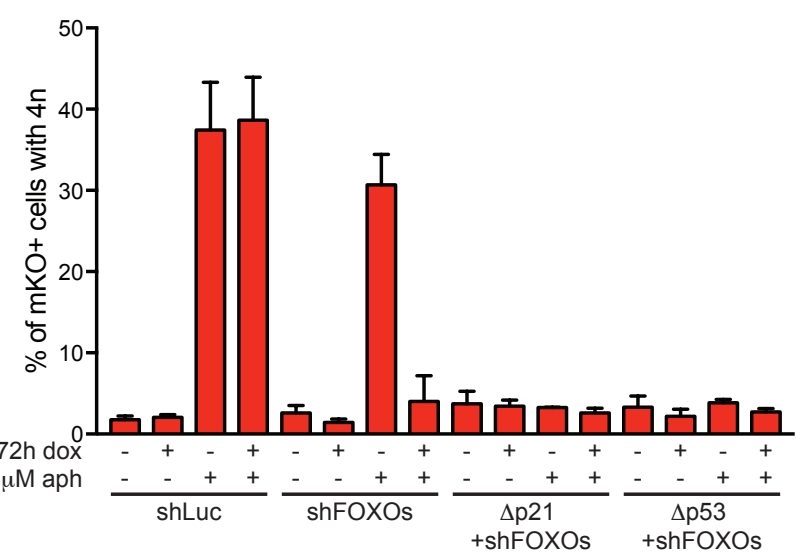

F

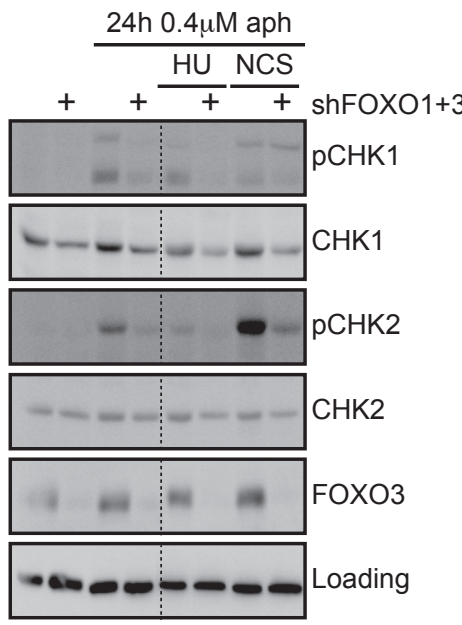

G

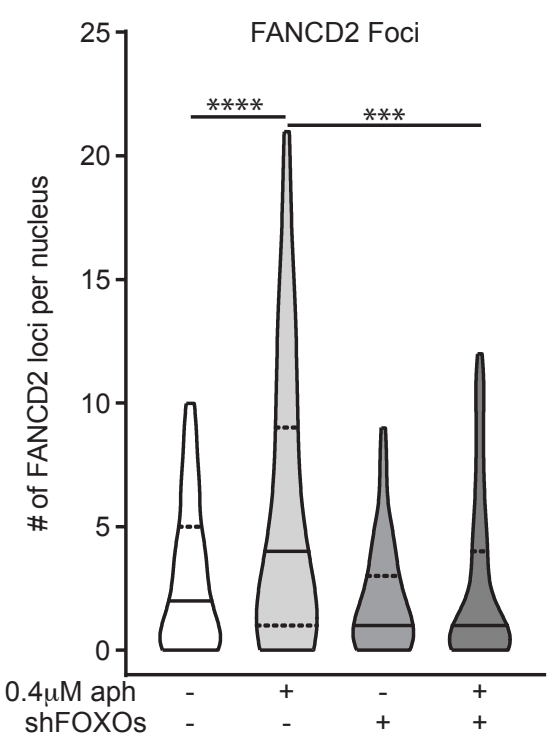

H

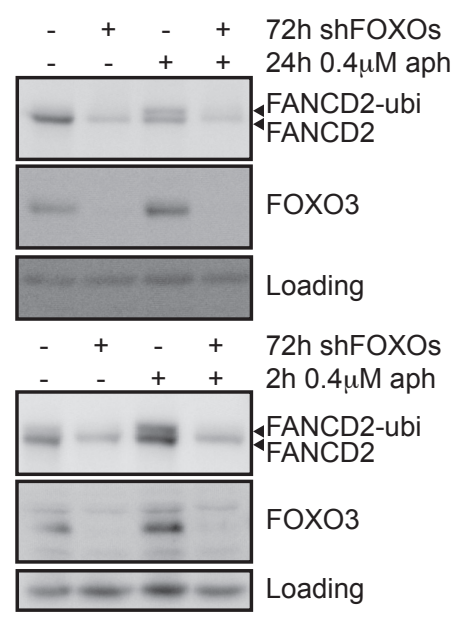

I

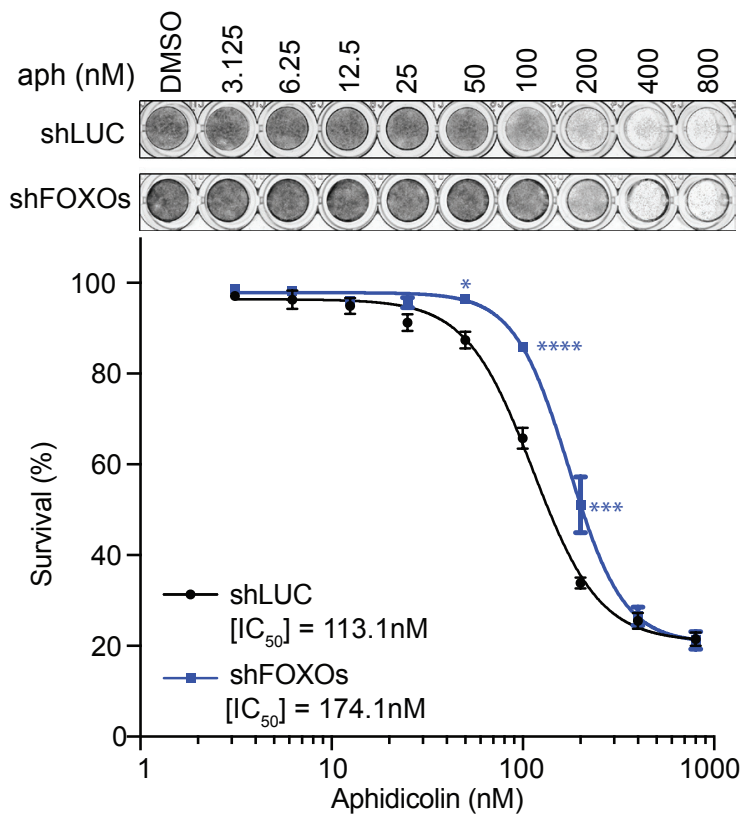




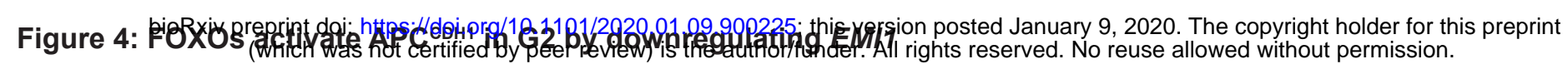

A

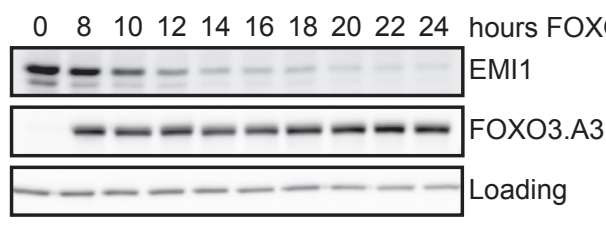

D

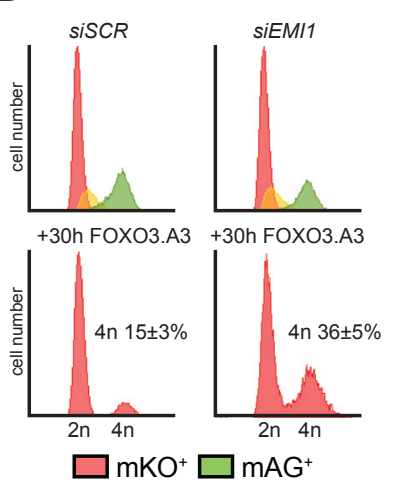

E

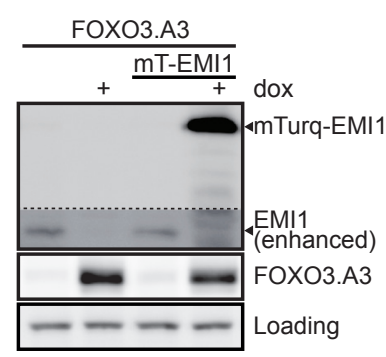

H

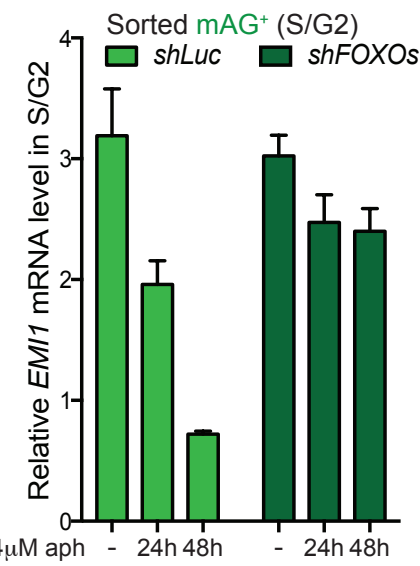

B

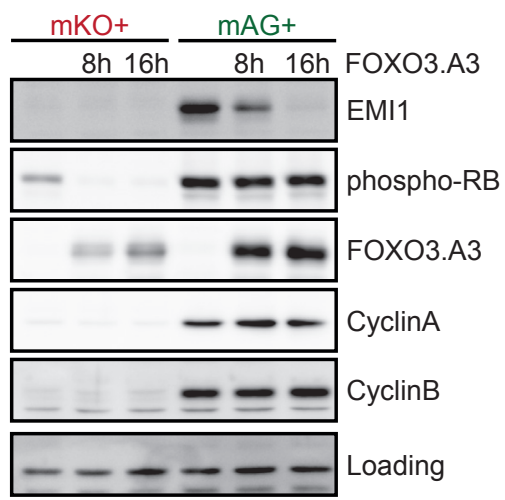

F

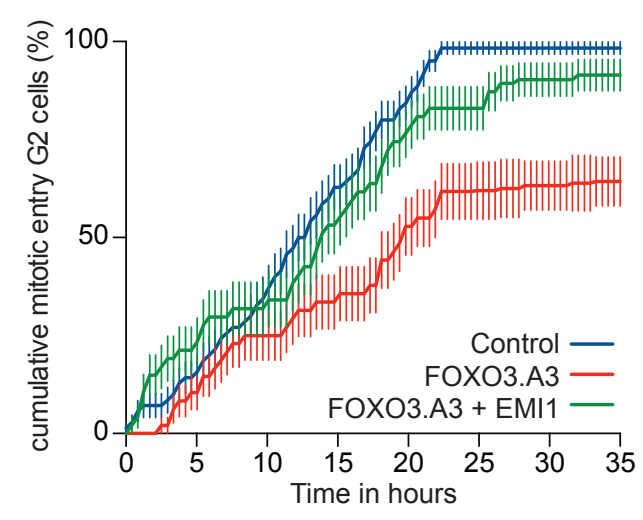

J
C

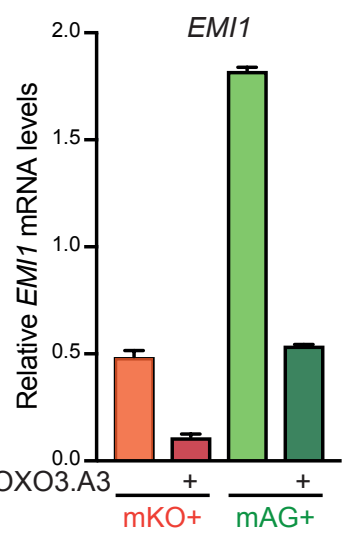

G

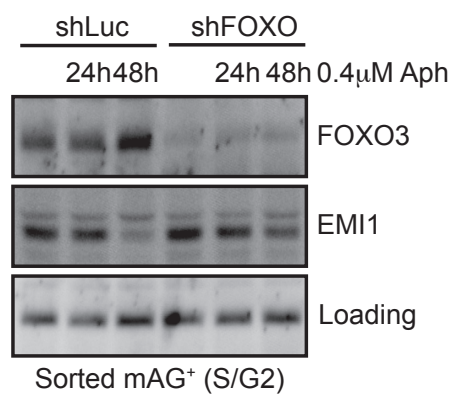

K

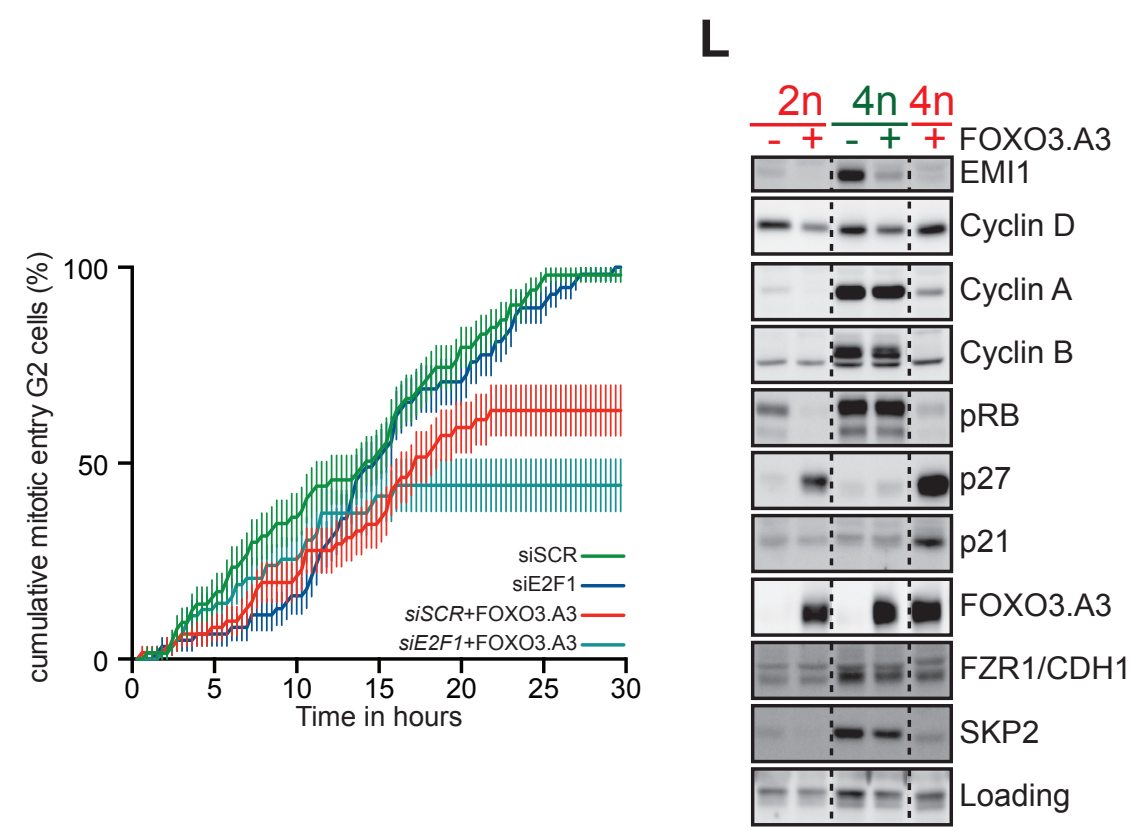

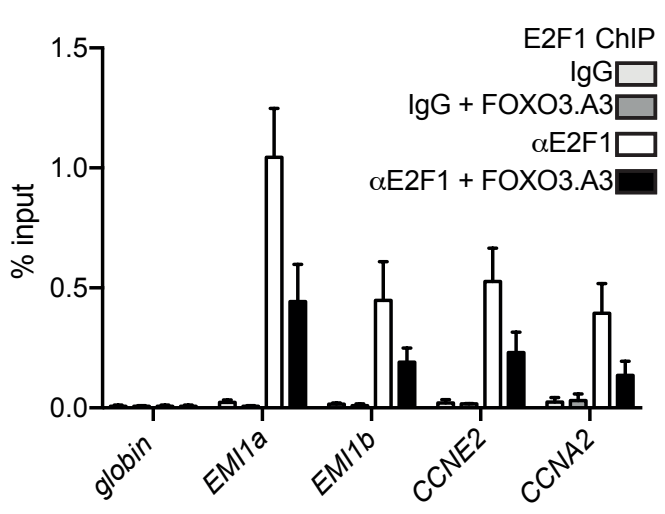

M
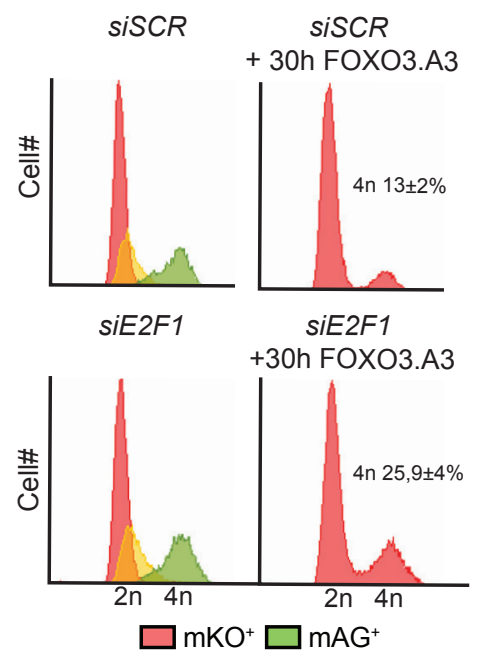

Normal cell cycle

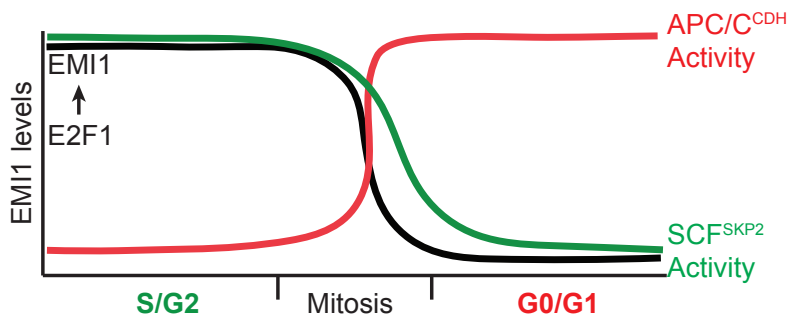

Replication stress

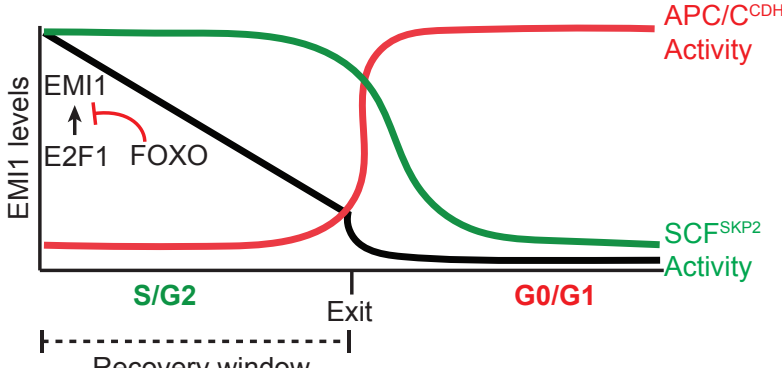

Recovery window 
Supplemenighaxifiereprint doi: https://doi.org/10.1101/2020.01.09.900225; this version posted January 9, 2020. The copyright holder for this preprint

A

aph $0.2 \mu \mathrm{M}$
$\mathrm{G} 2$ at $\mathrm{t}=15 \mathrm{~h}$

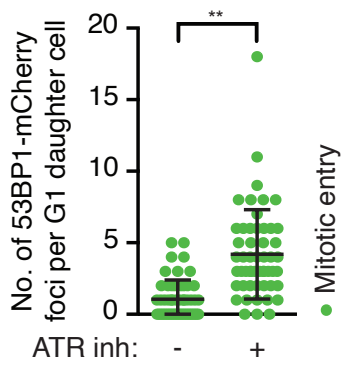

B

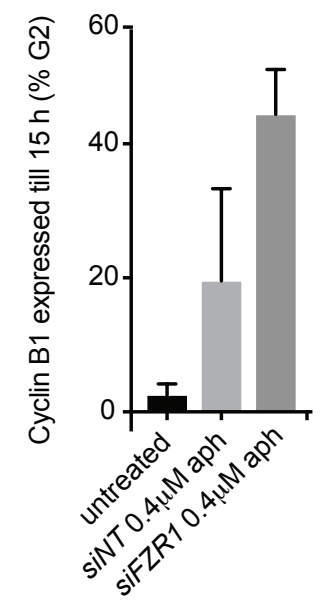

C

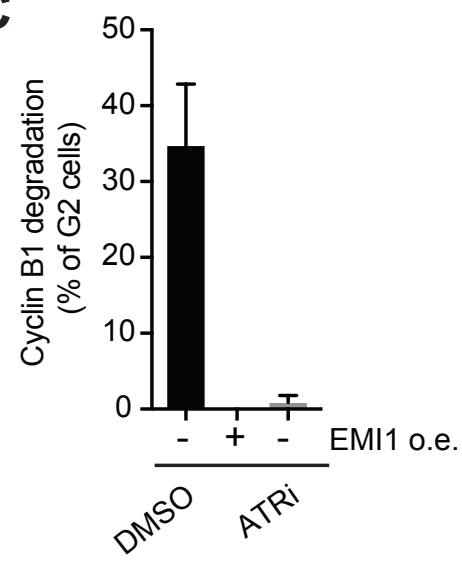




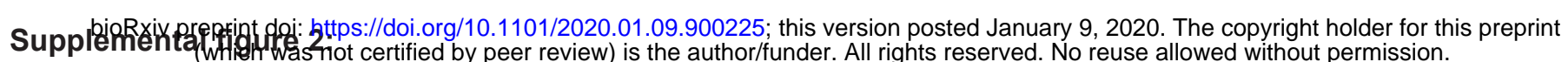

A

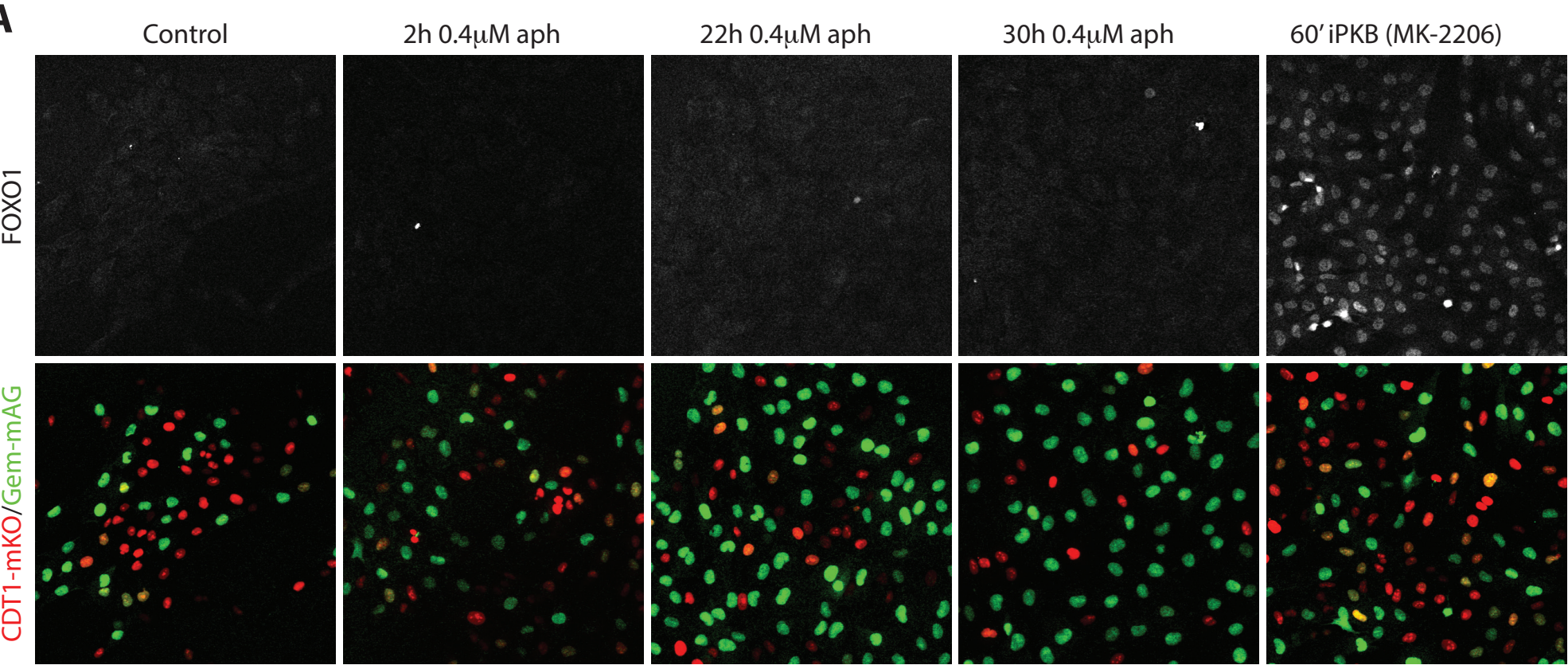

B

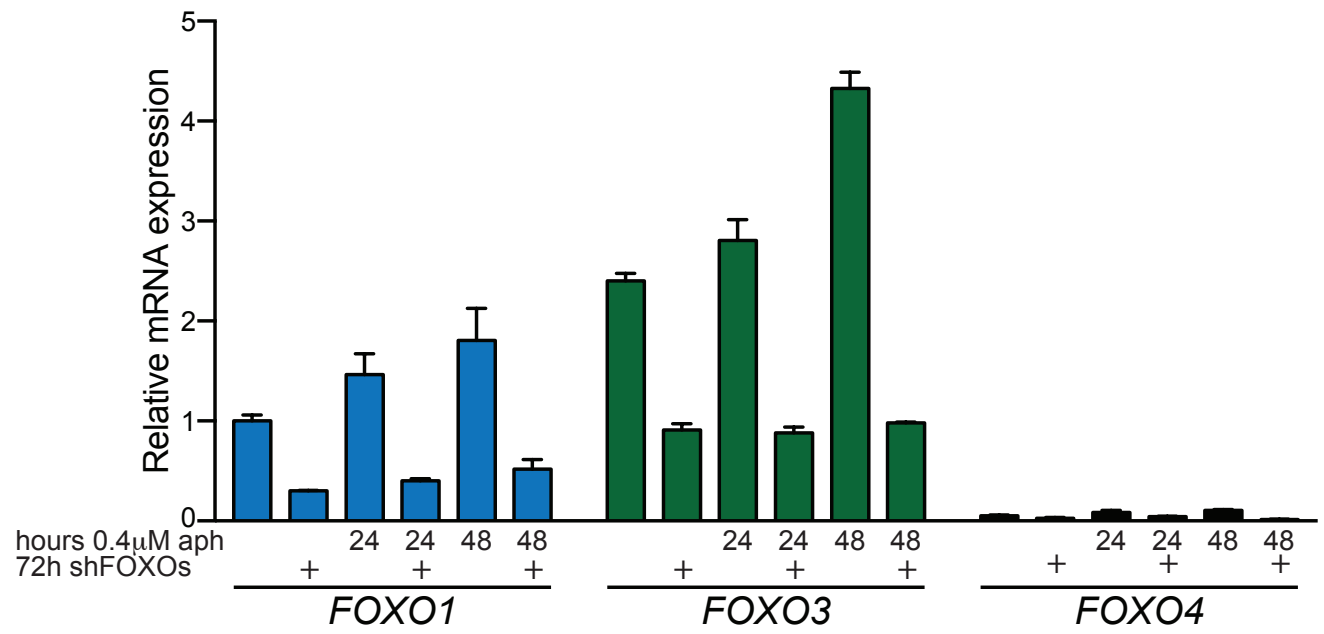

RPE-FUCCI

iFOXO3.A3

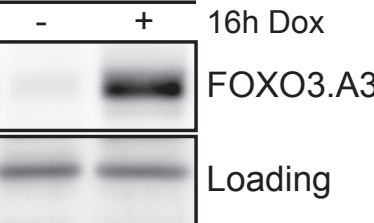


Sunplementaxizpreprint,doi: https://doi.org/10.1101/2020.01.09.900225; this version posted January 9, 2020. The copyright holder for this preprint Supplemental fig (w/fect was not certified by peer review) is the author/funder. All rights reserved. No reuse allowed without permission.

A

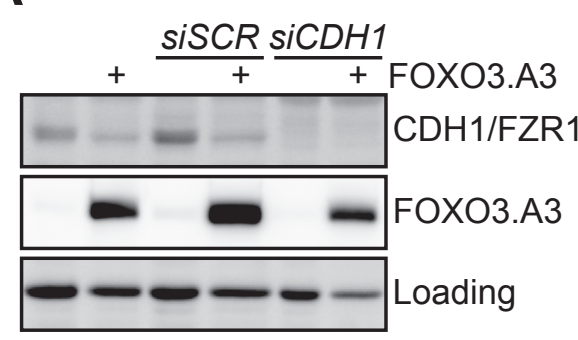

D

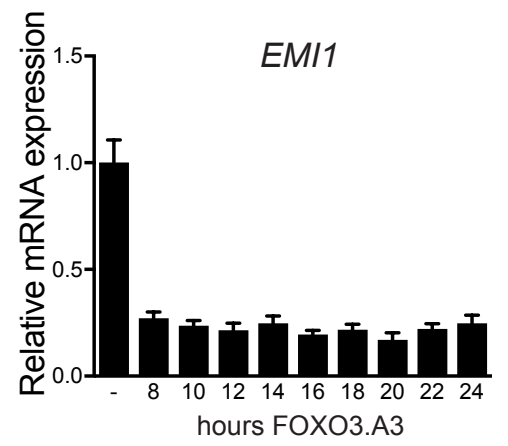

B
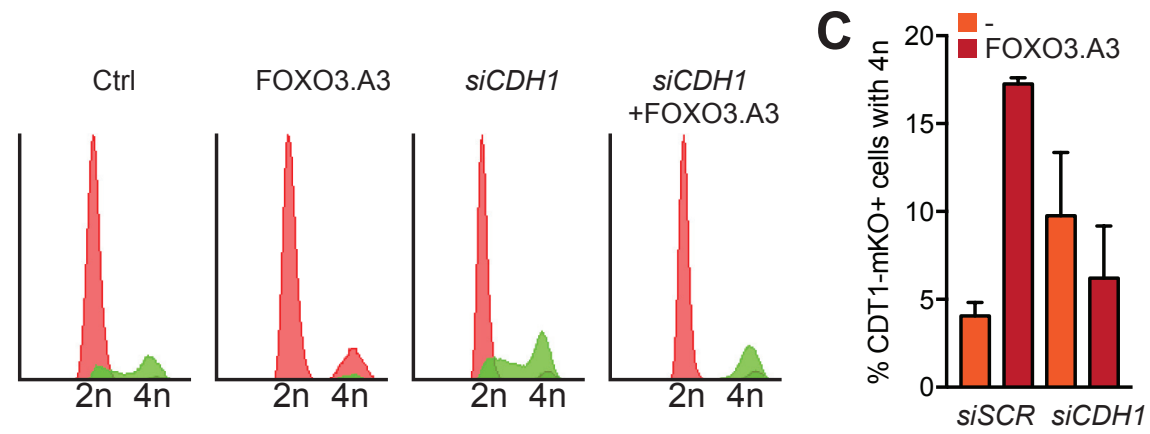

$\mathbf{E}$

$\mathbf{F}$
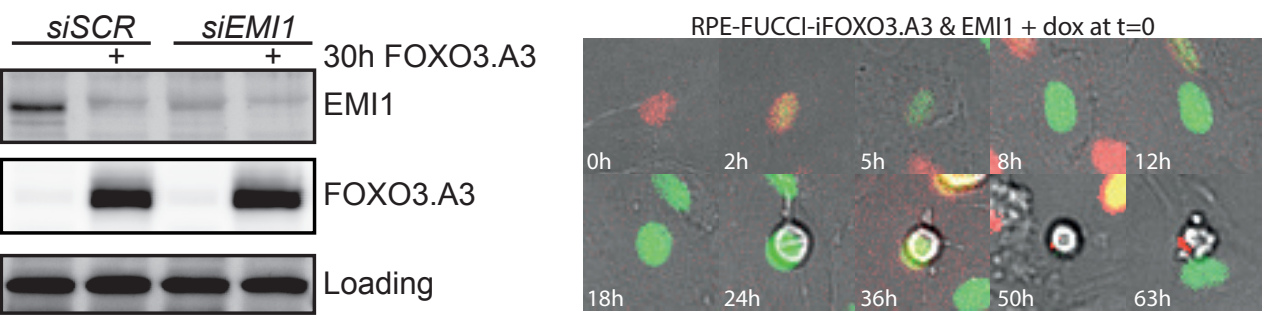


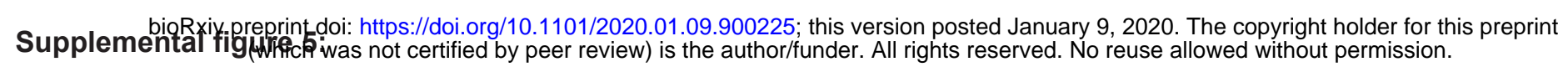

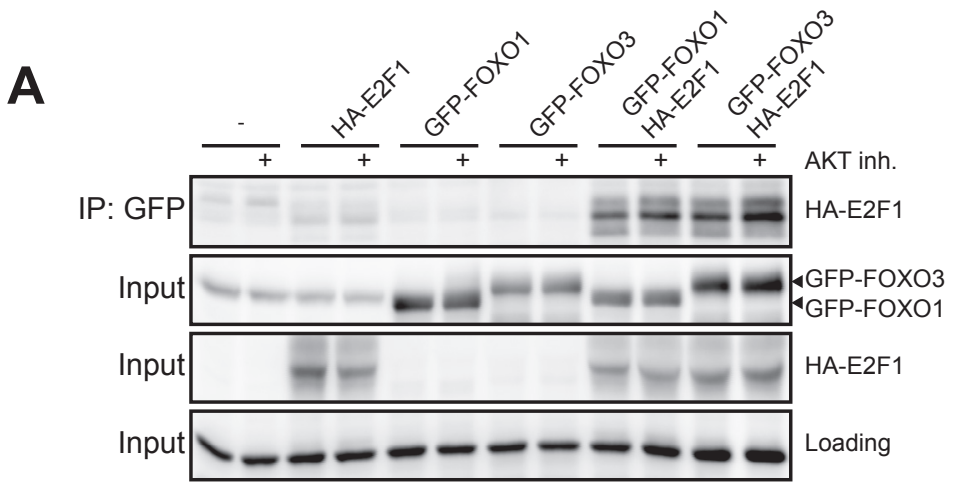

B

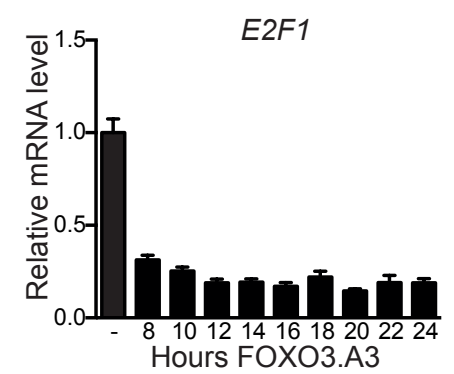

C

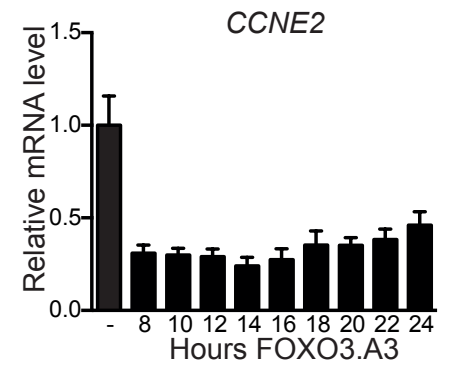

D

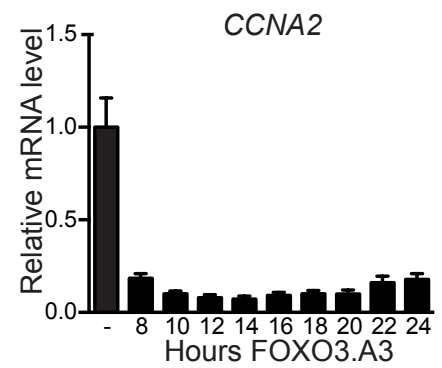

E

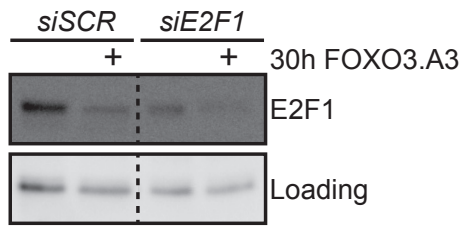

